Linköping Studies in Science and Technology

Licentiate Thesis No. 1687

\title{
DYNAMICS OF THE EARLY STAGES IN METAL-ON-INSULATOR THIN FILM DEPOSITION
}

\author{
Bo Lü
}

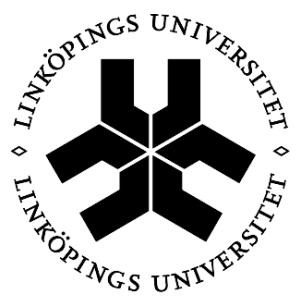

\section{Linköping University INSTITUTE OF TECHNOLOGY}

Nanoscale Engineering Division

Department of Physics, Chemistry and Biology (IFM)

University of Linköping, Sweden

Linköping 2014 
(C) Bo Lü

ISBN: 978-91-7519-192-8

ISSN 0280-7971

Printed by LiU-Tryck, Linköping, Sweden, 2014 


\section{Abstract}

Thin films consist of nanoscale layers of material that are used in many technological applications to either functionalize a surface or serve as parts in miniaturized devices. The properties of a film are closely related to its microstructure, which in turn can be tuned during film preparation. Thin film growth involves a multitude of atomic-scale processes that cannot always be easily studied experimentally. Therefore, different types of computer simulations have been developed in order to test theoretical models of thin film growth in a highly controlled way. To be able to compare simulation and experimental results, the simulations must be able to model events on experimental time-scales, i.e. several seconds or minutes. This is achievable with the kinetic Monte Carlo method.

In this work, kinetic Monte Carlo simulations are used to model the initial growth stages of metal films on insulating, amorphous substrates. This includes the processes of island nucleation, three-dimensional island growth and island coalescence. Both continuous and pulsed vapor fluxes are investigated as deposition sources, and relations between deposition parameters and film morphology are formulated. Specifically, the film thickness at what is known as the "elongation transition" is studied as a function of the temporal profile of the vapor flux, adatom diffusivity and the coalescence rate. Since the elongation transition occurs due to hindrance of coalescence completion, two separate scaling behaviors of the elongation transition film thickness are found: one where coalescence occurs frequently and one where coalescence occurs infrequently. In the latter case, known nucleation behaviors can be used favorably to control the morphology of thin films, as these behaviors are not erased by island coalescence. Experimental results of $\mathrm{Ag}$ growth on amorphous $\mathrm{SiO}_{2}$ that confirm the existence of these two "growth regimes" are also presented for both pulsed and continuous deposition by magnetron sputtering. Knowledge of how to avoid coalescence for different deposition conditions allows nucleation for metal-on-insulator material systems to be studied and relevant physical quantities to be determined in a way not previously possible. This work also aids understanding of the growth evolution of polycrystalline films, which in conjunction with advanced deposition techniques allows thin films to be tailored to specific applications. 


\section{PrefaCe}

This thesis is part of my $\mathrm{PhD}$ studies in the Nanoscale Engineering Division at the Department of Physics, Chemistry and Biology at the University of Linköping. The goal of my research is to contribute to the understanding of fundamental processes in the early stages of film formation of metals on insulators. This research is financially supported by the Swedish Research Council (Vetenskapsrådet, VR) and the University of Linköping. Research results are presented in three appended papers, following an introduction to the scientific field and research methods.

Bo Lü

Linköping, November 2014 


\section{APPENDED PAPERS}

1. Elofsson, V., Lü, B., Magnfält, D., Münger, E. P. and Sarakinos, K., "Unravelling the physical mechanisms that determine microstructural evolution of ultrathin VolmerWeber films" J. Appl. Phys. 116, 044302 (2014)

2. Lü, B., Elofsson, V., Münger, E. P. and Sarakinos, K., "Dynamic competition between island growth and coalescence in metal-on-insulator deposition", Appl. Phys. Lett. 105, 163107 (2014)

3. Lü, B., Münger, E. P. and Sarakinos, K. " Growth regimes during metal-on-insulator deposition using pulsed vapor fluxes " (2014) (manuscript in final preparation)

\section{AUTHOR'S CONTRIBUTION TO APPENDED PAPERS}

1. I contributed to the design of, performed the simulations and analyzed the simulation data. I wrote the part of the manuscript that concerns the simulations.

2. I contributed to the design of, performed the simulations and analyzed the simulation data. I conceived and developed the mathematical models proposed in the article. I wrote the manuscript.

3. I contributed to the design of, performed the simulations and analyzed the simulation data. I conceived and developed the mathematical models proposed in the article. I wrote the manuscript. 


\section{ACKNOWLEDGEMENTS}

I would like to thank my main supervisor Kostas Sarakinos for giving me the opportunity to work on a genuinely interesting subject. Your ambition inspires, and your drive to create a just and fair research environment makes working with you a joy indeed! Likewise I thank my co-supervisor Peter Münger. Without your guidance I would not have come this far. Thank you both for believing in me!

My fellow coworkers Viktor Elofsson, Daniel Magnfält and Sankara Pillay, from whom I have gained much support and wisdom in both work and life.

Former and present members of the Plasma and Coatings Physics Division, for the interesting discussions and laughter.

My family, for encouraging me to embark on this quest for knowledge.

To the one I loved... 


\section{TABLE OF CONTENTS}

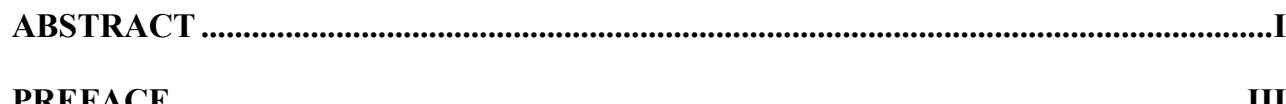

PREFACE ............................................................................................................................. III

APPENDED PAPERS........................................................................................................... V

AUTHOR'S CONTRIBUTION TO APPENDED PAPERS ..........................................................

ACKNOWLEDGEMENTS ............................................................................................. VII

1 INTRODUCTION...................................................................................................

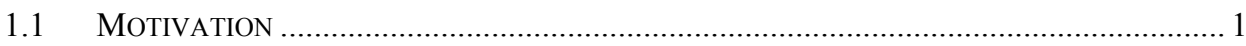

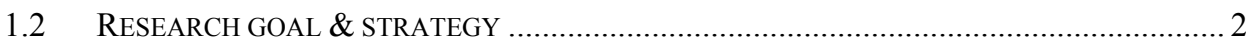

2 THIN FILM GROWTH .......................................................................................................

2.1 ATOMISTIC MODELS OF THIN FILM GROWTH ........................................................... 5

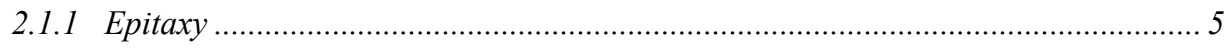

2.1.2 Nucleation and growth on amorphous surfaces ................................................. 11

2.1.3 Island coalescence ................................................................................................ 14

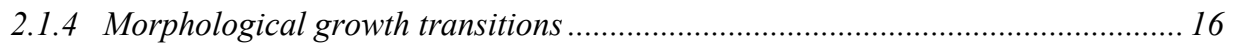

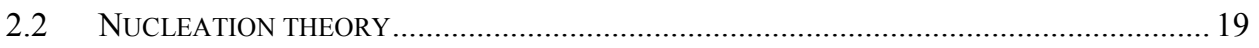

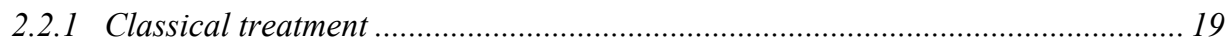

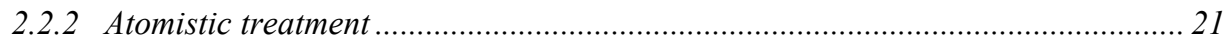

2.3 FILM GROWTH UNDER PULSED VAPOR FLUXES .................................................. 24

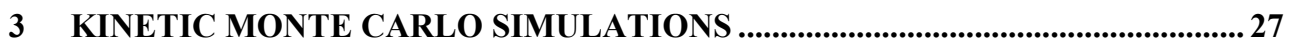

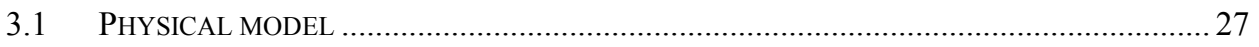

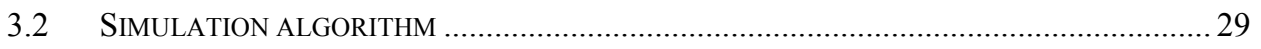

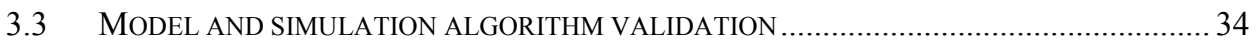

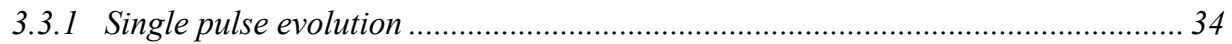

3.3.2 Island density and average size evolution in droplet growth ............................... 35

3.3.3 Treatment of secondarily nucleated islands ...................................................... 38 


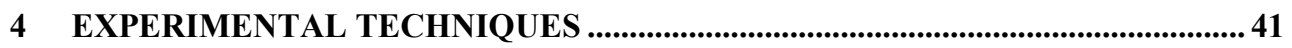

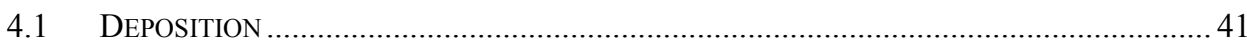

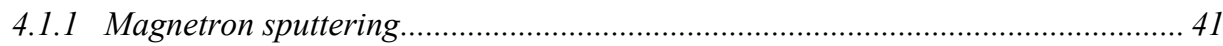

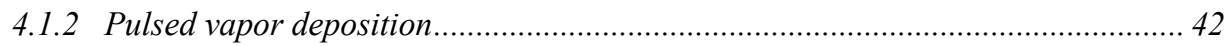

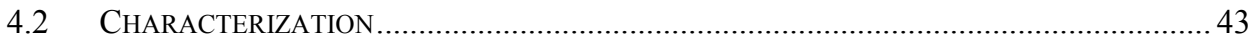

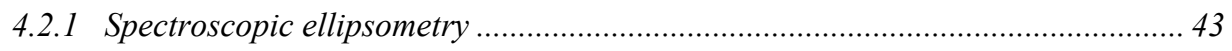

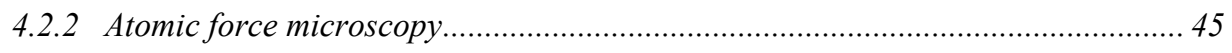

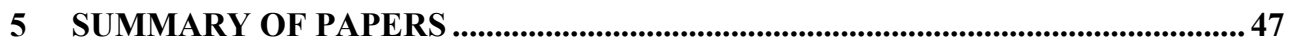

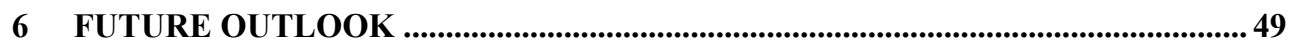

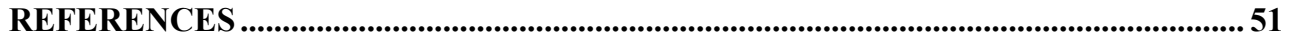

PAPERS 1-3 


\section{INTRODUCTION}

\subsection{Motivation}

Thin films refer to a layer of any material that has a thickness ranging from a single atomic layer up to several micrometers, and nanostructures refer to any material comprised of nanoscale particles. These objects have become an integral part of modern technology, with applications ranging from microelectronics to cutting tools. [1] Films are typically used to functionalize the surfaces of objects, for instance by making them scratch-resistant, corrosionresistant or simply more appealing to the eye. Exotic new materials can also be produced, as the small length-scales can force otherwise immiscible materials to form metastable alloys, or a film to adopt an entirely different atomic structure, imposed by the substrate that carries it. Due to quantum confinement effects, nanostructures may exhibit drastically different properties than its bulk counterpart such as a size-dependent band-gap or shape-dependent photocatalytic properties. [2-4]

Apart from the chemical composition, the properties of a thin film are also determined by its structure, or morphology, which in turn is a product of the growth process. Today, the majority of thin films are synthesized from the vapor phase, either chemically (chemical vapor deposition, CVD) or physically (physical vapor deposition, PVD). These processes are typically regarded as being far-from-equilibrium, as kinetic restrictions prohibit the system from reaching the minimum energy configuration as predicted by thermodynamics. [5,6] For instance, the ability for an atom to diffuse along the edge of an atomic step determines whether the step will grow smoothly or become jagged. Driven by technological advancement, much research has been conducted to catalogue and understand the different kinetic limits that occur in the formation of thin films and nanostructures, in an effort to learn how to control the growth process and produce materials tailored to specific applications. In the past 50 years, substantial advancement in the field of epitaxy ${ }^{1}$ has been made, owing to a large extent to the need for miniaturization in the electronics industry. [5,7,8] In particular, metal-on-metal homoepitaxy ${ }^{2}$ has been used as a model system due to the ability to clearly distinguish thermodynamic effects from kinetic effects on the growth morphology. However most applied thin film technologies are not homoepitaxial systems, in fact, in many cases they are not even epitaxial! On structurless, amorphous substrates such as glass, randomly oriented

\footnotetext{
${ }^{1}$ Growth of a crystalline layer on a crystalline substrate that retains the same crystallographic structure

${ }^{2}$ Epitaxy in the case the film and substrate are of the same material
} 
grains are nucleated and later reshaped by coalescence between islands. [9-11] This leads to a seemingly random distribution of grains separated by grain boundaries in the final, polycrystalline film, which is a fundamentally different structure than that encountered in epitaxially grown films. The presence of grain boundaries, size and shape effects of the grains can give rise to vastly different physical attributes as compared to bulk and single-crystalline materials. By finding patterns in the randomness, we may be able to gain a deeper understanding of the growth evolution of thin films deposited on amorphous surfaces. This will ultimately enable the smart design of a wide range of technologically important materials.

\subsection{RESEARCH GOAL \& STRATEGY}

At present, the early stages of film growth on amorphous surfaces are not entirely understood, thus the ability to make intelligently designed materials by varying the growth conditions is often rudimentary or based on trial and error. The goal of this thesis is to contribute to this understanding that is paramount to knowledge-based synthesis of thin films and nanostructures. In this work, I have concentrated my efforts to the study of metallic films grown from the vapor phase on amorphous substrates, using silver (Ag) deposited on silicon dioxide $\left(\mathrm{SiO}_{2}\right)$ as a model system. Industrially, this material system can be found in applications such as low emissivity windows, metallization in microelectronics and supported nanoparticles for photocatalysis. A kinetic Monte Carlo (kMC) growth simulation was developed to visualize the early and intermediate stages of film formation, and growth experiments were performed to provide information on the intermediate and late stages of the same. By combining the information gained from simulation results and both in situ and ex situ experimental analysis, new predictive models are theorized that can describe the film structural properties in the late stages (up to continuous film formation). These models are based on the effects of growth processes in both the early and intermediate stages, and begin to bridge the knowledge gap mentioned earlier.

Many of the different growth processes in the early and intermediate stages of film formation occur on different timescales. [2] For example, substrate diffusion occurs on the order of micro- to nanoseconds, while island coalescence occurs on the order of milliseconds to seconds. This can be used to our advantage if the deposition source is pulsed such that vapor arrives at the substrate in short bursts of controllable width and amplitude. In this way, different processes are affected independently and in different ways by vapor flux arrival and addition of material. [12-16] To this end, further understanding of the effects of pulsed vapor 
deposition sources (specifically, pulsed magnetron sputtering) is another primary focus of this work, hence a pulsed vapor source was used in the growth experiments and modeled in the kMC simulations as well. However, additional experiments and simulations of continuous vapor sources were also performed and investigated in order to differentiate the effects of pulsing on film growth from average deposition rate effects.

We begin by reviewing the fundamentals of thin film growth in Chapter 2. Here, physical models of the initial stages of different thin film growth modes are presented, from nucleation up to continuous film formation. The chapter is concluded with a presentation of mathematical treatments of thin film nucleation from both a thermodynamic as well as atomistic point of view. In Chapter 3, the physical model chosen for the kMC simulations is discussed, with a presentation of validation results, and the simulation code is described. Chapter 4 presents the different experimental techniques used in the appended papers, beginning with deposition techniques and followed by characterization methods. Finally, a summary of the appended papers is given in Chapter 5 and a brief discussion on the future prospects of the research topics in this thesis are discussed in Chapter 6. 
CHAPTER 1 : INTRODUCTION 


\section{THIN FILM GROWTH}

\subsection{ATOMISTIC MODELS OF THIN FILM GROWTH}

\subsubsection{EPITAXY}

Beginning with the most well studied case of thin film growth, epitaxy describes the growth of atomic layers on the surface of a crystalline substrate where new layers adopt the crystal structure of the substrate. This can be further separated into homoepitaxy, where the film and substrate are of the same material, and heteroepitaxy, where the film and substrate are of different materials. For the present work, understanding epitaxy not only provides a basic understanding of the atomistic processes that occur during film growth, but it is also relevant for polycrystalline film growth since homoepitaxial growth occurs on individual grains. In the following, the essential ideas of epitaxy close to thermodynamic equilibrium are described, followed by a short review of key kinetic effects in epitaxy far from equilibrium. As we are concerned with the growth of $\mathrm{Ag}$, most of the examples and references given deal with metalon-metal homoepitaxy, specifically face-center-cubic (fcc) metals such as copper, aluminum, lead or gold.

In 1958, Ernst Bauer classified the growth modes of epitaxy based on thermodynamic considerations. [17] Based on the balance between the surface free energies of the substrate $\left(\gamma_{S}\right)$, the deposit $\left(\gamma_{D}\right)$ and the interface between these $\left(\gamma_{I}\right)$, three distinct modes can be described. When $\gamma_{S} \geq \gamma_{D}+\gamma_{I}$, the deposit tends to "wet" the substrate, covering as much area with as little material as possible in order to maximize $\gamma_{D}$ and $\gamma_{I}$, leading to the Frankvan der Merwe growth mode commonly found in homoepitaxy. This condition may be satisfied in heteroepitaxial systems as well, but if there is a large difference between the lattice parameters of the substrate and the deposit (a large lattice mismatch), strain energy will begin to build up as the first few atomic layers wet the substrate. This strain energy contributes to $\gamma_{I}$, and very quickly the condition is reversed into $\gamma_{S}<\gamma_{D}+\gamma_{I}$. This leads to the formation of three-dimensional (3D) islands on top of the strained layers, as the reduced interface area minimizes $\gamma_{I}$. However for heteroepitaxial systems, it is more common that the condition $\gamma_{S}<\gamma_{D}+\gamma_{I}$ is valid from the outset and 3D islands form immediately on the substrate, leading to the Volmer-Weber growth mode.

The applicability of these classifications to modern thin film growth is limited, since most deposition techniques are far-from-equilibrium processes. [5] Nevertheless, it is common to 
find them designating different growth morphologies in the literature for sake of simplicity, even though the cause of the structure formation is inherently kinetic in nature. The basic idea of kinetic limitation is depicted in Figure 1. [18] A reaction $A+B \rightarrow C$ releases energy $\Delta H$ once equilibrium is established, and is thus thermodynamically favorable. However, the rate of the reaction is dependent on the magnitude of the activation energy barrier that must be surmounted. Thus, only reactants possessing sufficient thermal (i.e. vibrational) energy contribute to the formation of state $C$, while other reactants may become trapped in intermediate, "metastable" states such as $C^{*}$. In the figure, the reaction is exemplified by the shape relaxation of a small, irregularly shaped island A by mobile atoms B on its surface. This process develops positive energy upon reaching the shape of a hexagon due to surface energy minimization, but becomes kinetically limited at low temperatures and instead forms an isosceles triangle with truncated corners. The mechanisms associated with this particular process will be discussed later. Upon a small temperature increase, the reactants at $C^{*}$ can cross the remaining activation barrier from $C^{*}$ to $C$ and the triangular island reshapes to the fully stable, hexagonal shape.

In order to describe the kinetics of thin film nucleation, we begin with the fundamental process of single atom surface diffusion. In its simplest form, this can be described by atoms

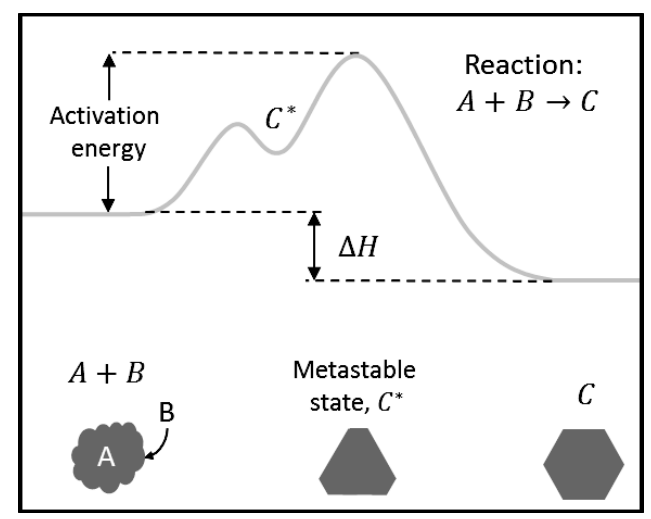

Figure 1. Schematic illustration of a reaction in terms of the energies involved, adapted from reference [18], and exemplified by the shape-relaxation of an atomic island. The activation energy is a a barrier that must be negotiated before the reaction can proceed and determines the rate at which the reaction takes place. $\Delta H$ is the energy gain when the reaction reaches equilibrium and $C^{*}$ is a metastable state, i.e. a local minimum on the potential curve that may trap the reaction if the energy of the reactants is insufficient to cross the activation barrier. 
"hopping" to nearest-neighbor $(\mathrm{NN})$ adsorption sites at a rate

$$
h=a^{2} v_{0} \exp \left(-E_{A} / k_{B} T\right)\left[m^{2} / s\right],
$$

where $a[m]$ is the distance between $\mathrm{NN}$ sites, $v_{0}[\mathrm{~Hz}]$ is the attempt frequency of an atom (typically $10^{12}-10^{13} \mathrm{~Hz}$ ), $E_{A}[\mathrm{eV}]$ is the activation barrier to overcome for a successful hop, $k_{B}[e V / K]$ is the Boltzmann constant and $T[K]$ is the temperature. Typically, $E_{A}$ is on the order of $0.1-1 \mathrm{eV}$ for most materials (compare to $k_{B} T \approx 0.026 \mathrm{eV}$ at room temperature, $300 \mathrm{~K})$. For surface diffusion, the diffusivity or diffusion constant $D=h / 4\left[\mathrm{~m}^{2} / \mathrm{s}\right]$ is typically reported in the literature. The specific path taken by a diffusing atom is strongly dependent on its local environment. The classically accepted model of a crystal surface, sometimes referred to as the step-edge-kink model, is schematically depicted in Figure 2. [19] A new layer begins to form on a flat, empty surface called a terrace. This layer is surrounded by edges, with corners forming where two edges meet. These edges may also be disrupted by kinks, which consist of single-atom-width displacements of the edge. Also belonging to this category are inverted corners and "holes" in an edge, both counted as special cases of kinks. The relative ease for an atom to diffuse in each of these local environments is the underlying cause of kinetic limitation during growth, and can be estimated on the bases of a simple bond counting scheme. [7] An atom diffusing on the terrace feels only the attractive forces from the atoms beneath it, thus it moves rather easily. In contrast, an atom moving along an edge feels forces from both the atoms below it as well as in the edge, thus it becomes more difficult to move in this environment. Leaving the edge for the terrace requires breaking of bonds and is further more difficult, and likewise leaving a kink site for an edge or terrace is the most difficult move an atom can make. By tuning deposition parameters such as the temperature and deposition rate, these different diffusion mechanisms become successively "activated", i.e. made to occur at a statistically relevant rates, allowing intentional control of the growth morphology. [5]

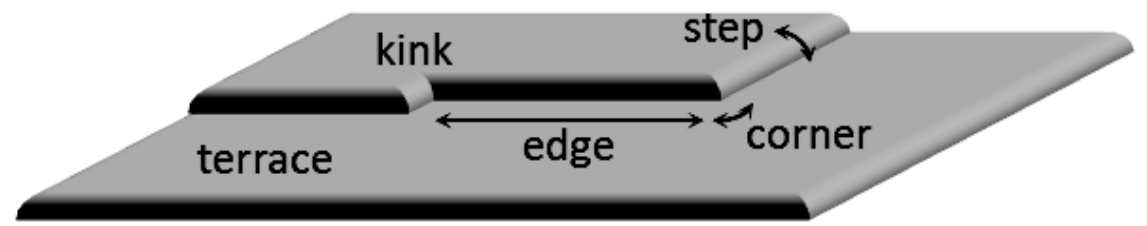

Figure 2. Illustration of different local environments that can be found on an atomic scale, based on the step-edge-kink model. 
So far, only atomic motion within a single horizontal plane (intralayer diffusion) has been described. In contrast, interlayer diffusion involves atoms crossing between parallel planes by descending at steps, i.e. the atomic positions on top of an edge (see Figure 2). This gives rise to two cases of edge attachment: atoms approaching an edge from the terrace attach at an ascending step, while atoms that cross the step from above attach to the edge at a descending step. The kinetics of step crossing cannot be estimated by bond counting, as in the transition state of the crossing, an atom is neither bound to the upper nor the lower terrace. This leads to an under-coordinated and unfavorable high energy state, which produces an explicit barrier to step crossing, known as the Ehrlich-Schwoebel barrier after its discoverers [20,21]. The potential landscape around step illustrating the Ehrlich-Schwoebel barrier is schematically shown in Figure 3. If this barrier is prohibitively large, films may grow three-dimensionally as atoms collect on top of pre-existing layers, nucleating successive layers before the previous one has filled out completely. This is known as "mound formation", and may occur even when the Ehrlich-Schwoebel barrier does not prevent step-crossing completely. In this case, atoms tend to be repelled at descending steps, leading to increase attachment at ascending steps which creates a diffusional "bias" in the "uphill" direction. These processes are collectively known as kinetic roughening, [22-26] and are typically encountered at low growth temperatures. Specifically, below a material-specific temperature called the kinetic roughening temperature, it is no longer possible for the film to grow two-dimensionally. [27]

If the film material possesses an intrinsically small Ehrlich-Schwoebel barrier or a high growth temperature is used to facilitate negotiation of this barrier, purely two-dimensional

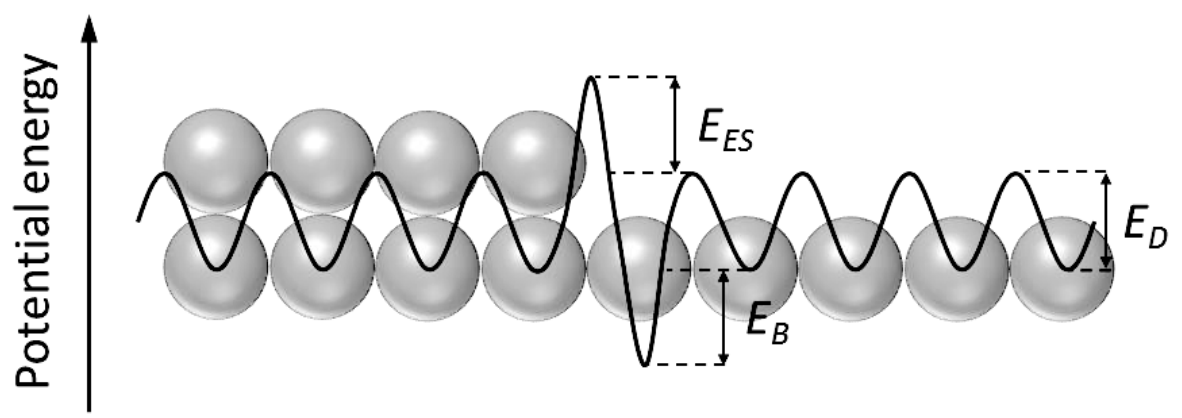

Figure 3. Schematic illustration of the potential landscape in one dimension at a monatomic step. $E_{D}$ is the diffusion barrier on a flat terrace, which is identical both above and below the step. $E_{B}$ is the binding energy to lateral atoms in the step edge, which gives rise to a minimum energy state at the edge. $E_{E S}$ is the Ehrlich-Schwoebel barrier associated with step crossing. 
growth modes arise. For example, if additionally terrace diffusion is fast relative to the deposition rate, a new layer tends to form by the growth of individual edges in their normal directions (step-flow growth). At intermediate diffusion rates relative to the deposition rate, nucleation of isolated, single-atom-layer islands occurs on the terrace and a new layer is formed by the merging of these (layer-by-layer growth). The in-plane shape of islands can in turn be controlled by the rate of edge diffusion, with dendritic ${ }^{3}$ islands forming if this is slow and compact islands forming for facile edge diffusion. [28-31] Depending on the film and substrate materials, the relative ease of corner crossing may also create variations in the compact island shapes, from triangles (as was described in Figure 1 for fcc (111) substrates) to aligned nano-rods (for fcc (110) surfaces). [30,32-34] Similar to steps, the kinetics of corner crossing cannot be estimated on the basis of bond counting, since the distance traveled by the mobile atom exceeds the NN distance.

However as with intralayer diffusion, the local environment around a step has proven crucial in determining the actual barrier for descent, hence the roughening transition temperature is also specific for different crystal surfaces, or facets. [35,36] For instance, it has been shown that the barrier changes depending on the height of the step (essentially, this accounts for diffusion between two separate facets), [37-41] the availability of kinks and corners or the crystallographic orientation of the underlying step edge [42-45] or the presence of an edge intersecting the step [46,47]. Using this knowledge, it was possible to explain what is known as "re-entrant layer-by-layer growth", where two-dimensional growth reappears for temperatures well below the kinetic roughening temperature. $[48,49]$ This was attributed to the abundance of kinks at the edges of dendritic islands (which are predominantly formed due to restricted edge diffusion) that in turn enhanced the amount of step crossing. Furthermore, extensive molecular dynamics (MD) simulations revealed that "exchange" events are highly likely to occur at steps, where an atom atop the step trades places with an atom in the step by pushing the latter out of the edge and onto the terrace. $[41,43,50]$ In fact for many materials, this has been shown to be the dominant step crossing mechanism, as the corresponding activation barrier is found to be much smaller than for direct hopping over the step. Theoretically, exchange processes exist for all of the diffusion scenarios discussed so far, e.g. corner exchange and even edge exchange or terrace exchange (a substrate atom "pops up" and the diffusing atoms takes its place), though they are typically found to be less favorable than

\footnotetext{
${ }^{3}$ So called due to their reminiscence of the crown or roots of a tree. The terms "ramified" or "fractal" are used synonymously in the literature.
} 
conventional NN hopping. [51-55] Finally, negative Ehrlich-Schwoebel barriers have also been suggested, in the sense that atoms are attracted towards descending steps instead of being repelled at these. [56,57] Naturally, this has implications for mound formation, with kinetic roughening occurring above some critical temperature instead of below.

One may wonder whether atoms ever diffuse upwards at steps. Conventional thinking suggests this is an extremely difficult diffusion process, as the total activation barrier as shown in Figure 3 is comprised of lateral atomic bonds, the terrace diffusion barrier and the Ehrlich-Schwoebel barrier. Even at high temperatures, this is deemed unlikely or at least statistically irrelevant when compared to all other diffusion processes. However, particular examples of true upwards diffusion do exist under certain conditions, such as for $\mathrm{Al}(110)$ homoepitaxy, where metastable huts that are tenfold higher than the average film thickness can only be formed if upwards diffusion is possible. [40,58,59] Other authors have theorized that upwards diffusion might be facilitated by step "permeability. [60-64] Normally, step edges are considered stable "sinks" for adatoms due to the formation of lateral bonds. As such, adatoms that have attached are only able to move along the edge, but cannot leave the edge for the terrace, and this would be the case irrespective of whether an adatom approached the edge from the ascending or descending directions. In the theory of step permeability, the lateral bonds are considered to be very weak, and adatoms that reach a step do not necessarily get trapped along the edge. This then allows them to either move away from a descending step after the descent, or to cross an ascending step in the upwards direction. Furthermore, the formation of low-index facets (e.g. $\{100\},\{110\}$ or $\{111\}$ on a cubic lattice) means that upward diffusion could be as easy as terrace diffusion. Assuming a simple shape for an island (see Figure 4), an atom deposited (or diffused) onto a side facet may eventually reach the edge of the top facet, and by overcoming the facet-facet barrier, it will have effectively contributed to upwards diffusion. This mechanism has been shown for polycrystalline growth of certain materials on amorphous surfaces, and will be discussed in the next section. $[10,65]$

Of course, the final remarks in the last paragraph beg the question, how realistic is deposition onto the side of an island, such as depicted in Figure 4? The answer to this is related to the concepts of "funneling" and "steering". Atoms deposited at a step edge are in an unstable state (equivalent to the transition state of step crossing by hopping), and the atom will fall in some direction; either down the step (downwards funneling ${ }^{4}$ ) or onto the top terrace (upwards

\footnotetext{
${ }^{4}$ This has also been theorized to contribute to the re-entrant layer-by-layer growth mode discussed earlier. [7]
} 


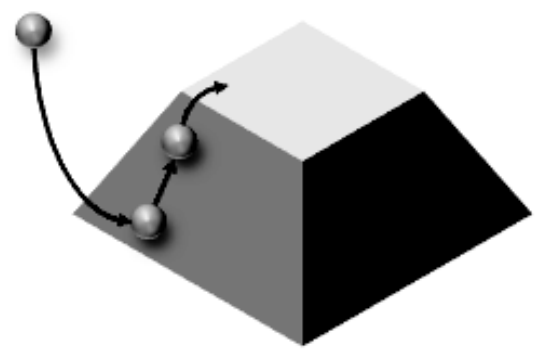

Figure 4. Illustration of the effect of steering, which bends atoms approaching a surface from the vapor phase towards perpendicular incidence to a local surface. Diffusion up a flat side facet followed by subsequent facet-facet crossing is shown as well.

funneling). [7,56,66-68] In this way, an atom deposited onto a side facet will be funneled downwards and may even reach the base of the island. However, if the incident atom does not possess enough kinetic energy, it will simply land or be funneled to some position on the side facet; a situation that has been dubbed "restricted downwards funneling". This is in turn enhanced by the "steering effect", where the trajectory of atoms approaching the film surface will tend to bend towards normal incidence to the local surface orientation. [7,69-71] Funneling has become recognized as an important structure formation mechanism in thin film epitaxy, as the slope of mounds formed at low temperatures is generally the result of a balance between downwards and upwards funneling, and the uphill diffusional bias cause by the Ehrlich-Schwoebel barrier. [7,56,67,68,72-76]

\subsubsection{NUCLEATION AND GROWTH ON AMORPHOUS SURFACES}

Metal films grown on amorphous surfaces are often classified to the Volmer-Weber growth mode since the difference in surface free energies of the film and substrate are typically large. Thus, in thermodynamic equilibrium, these films would grow by forming three-dimensional islands which grow together to form a continuous film. [22,77] After the initial nucleation, islands grow laterally until they reach a critical size, whereupon successive atoms attaching at the edge are forced upwards in order to form the second layer. The second layer then grows in a similar fashion, using the first layer as its substrate, and begins to force atoms upwards upon reaching its critical size. Similar critical sizes exist for higher layers as well, and the island as a whole effectively grows three-dimensionally. [22] This description is first and foremost based on the fact that film atoms bond weakly to the substrate, such that atoms in the island edge would rather minimize their potential energy by jumping up one layer, trading substrate 
bonds for stronger bonds with the atoms of the first atomic layer. This minimizes the interface between film and substrate, effectively contracting the base area of islands and lowering the free energy of islands as discussed in the beginning of section 2.1.1.

For some film-substrate combinations, the formation of low-index side facets on the islands that begin from the substrate level allows the lateral growth of three-dimensional islands to proceed by vertical layer-by-layer growth. $[10,65,78]$ This was shown for co-deposited aluminum-tin (Al-Sn) films grown on amorphous carbon (a-C), $\mathrm{SiO}_{2}$ and mica as well as epitaxially on sodium chloride $(\mathrm{NaCl})$. On mica and $\mathrm{NaCl}$, the effect was indicated by the segregation of Sn to the top of Al-Sn islands, which indicated that new layers formed at the base of islands and grew upwards. In contrast, growth on a-C and $\mathrm{SiO}_{2}$ showed segregation of Sn towards the base of the islands, indicating instead that new layers formed from the top of the islands and grew downwards. These two mechanisms are schematically shown in Figure 5. However, in reference [65] where these growth modes are presented, it is clearly stated that these models are only valid for growth temperatures above $30 \%$ of melting temperature of the film material(s). Thus the formation of vertical side facets may be justified on the basis of facile surface energy minimization.

When deposition occurs at temperatures well below the melting temperature of the film, the high activation barriers typically associated with upwards step crossing limits energy minimization, and the formation of side facets is hindered. Instead, the stepped surface of a growing island produces hemispherical cap-shaped particles, and a theory of nucleation and growth on amorphous surfaces based on kinetic limitations is required to explain this morphology. [6,11,79] Atoms are deposited onto a flat surface where they diffuse around randomly until they become trapped at substrate steps and defects or encounter another adatom. In the last case a dimer is formed in a random orientation (relative to other dimers), and if another adatom impinges the dimer, a trimer is former and so forth. Depending on the

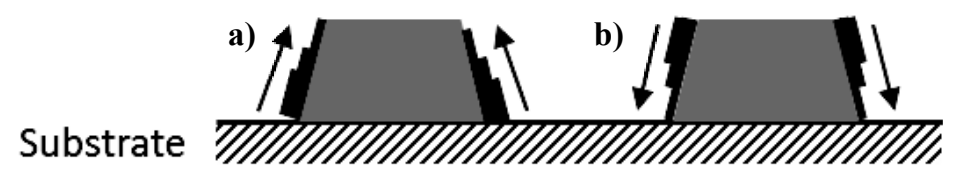

Figure 5. Illustration of the two growth mechanisms of polycrystalline islands as describe in [65]. Islands grow by forming new layers on its sides, either a) beginning from the bottom up or b) beginning from the top down. 
film and substrate materials, a dimer may constitute a stable nucleus around which an island begins to grow. The nucleus is stable in the sense that it is assumed to be immobile and the nucleation irreversible, allowing the definition of a critical nucleus size $i^{*}$ which for stable dimers is equal to one (for trimers, $i^{*}=2$ and so on). Initially, these newly nucleated islands will grow very little in the vertical direction, since second layer nucleation and thus vertical growth is driven by a combination of direct deposition onto islands and subsequent blocking of step crossing by the Ehrlich-Schwoebel barrier. At this stage, the islands are too few and too small to capture a sufficient amount of the deposited flux; most atoms land on the bare substrate and diffuse to the edges of pre-existing islands, thus contributing to their lateral growth (see Figure 6). It is this bias towards lateral growth that prohibits islands from attaining an equilibrium contact angle to the substrate.

A complimentary explanation for this bias can be found by comparing the rate of deposition to the rate of upwards step crossing for a given growth temperature. [6] An atom A at an island edge would like to cross the step upwards to find a more energetically stable site. If the deposition rate is fast enough, atom $\mathrm{A}$ will be joined by atoms $\mathrm{B}$ and $\mathrm{C}$ (which also attach to the island edge) before it can make its move. In this way, atom A becomes stabilized as it now forms a new "layer" in the island edge together with atoms B and C, and it is no longer more favorable for it to cross the step upwards. Since this is repeated for the new atoms B and C, the island grows predominantly in the lateral directions.

As the islands become larger, direct capture of atoms from the deposition flux becomes more effective and they begin to grow vertically as well. Eventually, the initial islands become

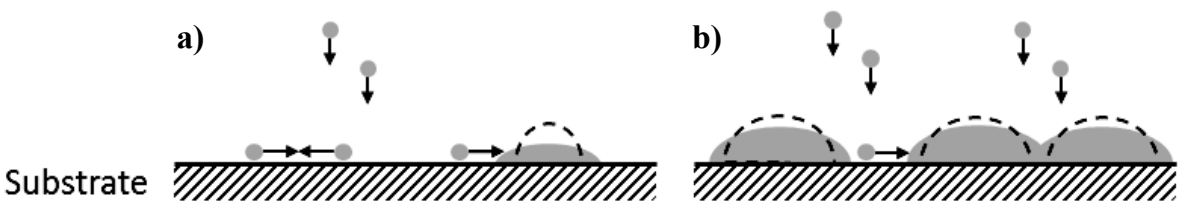

Figure 6. Illustration of the polycrystalline nucleation and growth mechanism described in [79]. a) Atoms diffusing on the surface agglomerate into islands and contribute to their lateral growth. Very little vertical growth occurs as the islands are too small to catch a sufficient amount of atoms from the vapor, which leads to island shapes that deviate from their equilibrium shapes (dashed lines). b) In later stages, islands begin to catch atoms from the vapor but due to the initially stinted vertical growth are still not able to reach their equilibrium shapes fully. Island-island impingements also begin to occur as the islands grow closer to each other. 
large enough in both size and number that subsequently deposited atoms are more likely to land on or diffuse to pre-existing islands rather than finding another adatom. This leads to a rapid decrease in the nucleation rate and consequently rapid increase in the island growth rate. Simultaneously, islands begin to impinge each other due to their proximity, and coalescence occurs.

\subsubsection{ISLAND COALESCENCE}

The process of coalescence involves the merging of two single-crystalline islands into a single, larger single-crystalline island. Depending on growth conditions and properties of the film material, island mobility may cause coalescence to occur earlier as well, [2,80-83] a process which has been termed "Smoluchowski ripening" to separate it from conventional growth, or static coalescence. For very small islands (up to a few atoms in size), coalescence likely resembles more a nucleation event, occurring in essence instantaneously be rearrangement of periphery atoms. For larger islands, the classical view of coalescence is based on surface migration of atoms driven by differences in curvature. Areas of great curvature in the neck that forms between two islands are preferentially filled out by atoms diffusing along step edges and descending from higher atomic layers (see Figure 7). Once the neck has been filled, surface energy minimization brings the newly formed island to its equilibrium shape by the same atomistic processes. In the absence of deposition, material must be detached from kinks and steps in order for coalescence and shape equilibration to progress. [84-89] Thus, if the temperature is too low, coalescence will be hindered due to the lack of mobile atoms. In this sense, the deposition source is of utter importance to enable fast coalescence at temperatures far below the melting temperature of the film. [5,6]

Coalescence by curvature-driven surface diffusion is often termed "liquid-like", as early investigators observed facetted islands in epitaxial growth studies being rounded upon contact and merging very rapidly, in a process that resembled melting. [6,90,91] However, transmission electron microscopy (TEM) and diffraction (TED) experiments have consistently shown that the merging islands remain solid (crystalline) throughout the coalescence process, and thus are not actually being melted; $[6,87,92,93]$ the turbulent and rapid mass transport on the surface only gives that impression. Care should be taken though, as the melting temperature is known to decrease for decreasing particle size. [88,94-97] Liquid-like coalescence has long been treated in the same as the sintering of two spheres by surface diffusion in metallurgy. [98-102] An early mathematical treatment of the time-dependent ratio between the neck width $\chi$ and sphere radius $r$ was given by Kuczynski as [100] 
a)

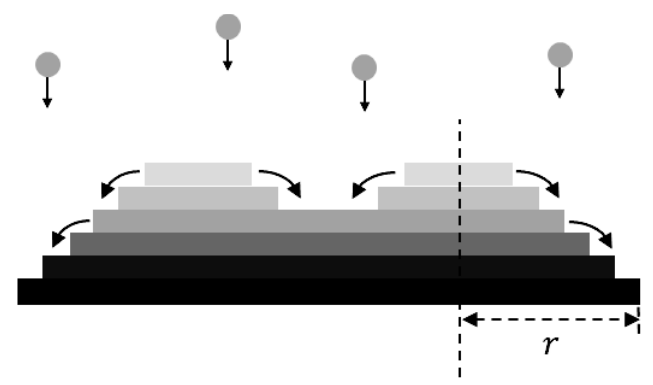

b)

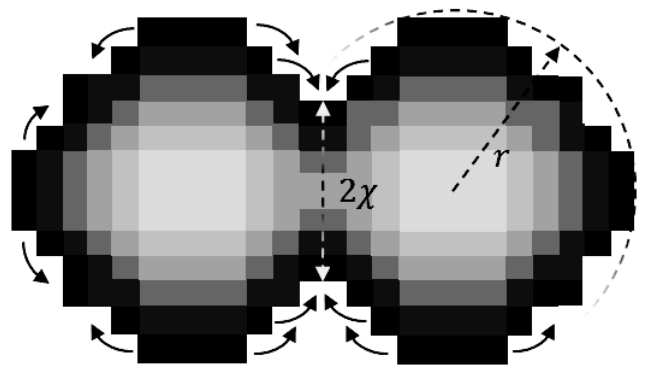

Figure 7. Illustration of the coalescence process between two islands. a) Atoms deposited on top of the islands descend at step edges, with the net effect of vertically filling out the neck region between islands. b) On each atomic layer, atoms diffuse along the edge towards areas of high concave curvature (equivalently higher coordination). Also, the parameters $r$ and $\chi$ used in Eq. 2 are indicated.

$$
\chi^{7} / r^{3}=\left(56 \sigma a^{4} / k_{B} T\right) D_{S} n_{s} t,
$$

where $\sigma[\mathrm{eV}]$ is the specific surface energy, $a[\mathrm{~m}]$ is the lattice spacing ( $a^{3}$ the atomic volume), $D_{S}\left[\mathrm{~m}^{2} / \mathrm{s}\right]$ is the self-diffusivity of the film and $n_{s}$ the concentration of atoms on the surface. The corresponding quantities of $\chi$ and $r$ in the case of island coalescence is indicated in Figure 7. A similar function was later described for the time-dependence of a complete coalescence process, from neck formation to shape relaxation, as [101]

$$
t_{\text {coal }} \approx r^{4} / B[s],
$$

where the parameter $B$ is based on the work of Nichols and Mullins, who derived [99]

$$
B=D_{S} \gamma \Omega^{2} \rho_{A} / k_{B} T\left[m^{4} / s\right] .
$$

In Eq. $4, \gamma\left[\mathrm{eV} / \mathrm{m}^{2}\right]$ denotes the isotropic surface energy, $\Omega\left[\mathrm{m}^{3}\right]$ is the atomic volume and $\rho_{A}\left[1 / m^{2}\right]$ is the planar density of atoms. Assuming a simple cubic lattice, which has $\rho_{A}=1 / a^{2}$, while $\Omega=a^{3}$, the product $\Omega^{2} \rho_{A}=a^{4}$. It can then be seen that Eq. 2 and Eq. 3 are nearly identical (save for a numerical factor in Eq. 2 ) for $\chi=r$, i.e. when the coalescence is complete and an in-plane circular geometry is recovered. 
Early studies of the morphological evolution of water droplets condensed on a window revealed a type of pattern formation driven by coalescence that can also be found in the nucleation and growth of metal thin films on amorphous substrates. Large droplets tended to cannibalize smaller ones, leaving a pattern where larger islands are well separated from each other, and smaller islands occupy the spaces between them. At this stage, the relative distribution of island sizes is maintained for a sustained period of time, leading to a "selfsimilar" growth regime. Extensive works on the scaling behavior of the island size distribution (ISD) in this growth regime have been performed, [75,103-108] though due to lack or relevance to the present work, they will not be presented here. As island growth and coalescence progress, areas of the substrate once covered by islands are "denuded" due to the redistribution of material in the coalescence process. New islands begin to nucleate on these denuded areas, forming a second generation of islands and breaking the self-similarity of the previous growth regime. In the condensation of water, successive generations of droplets will also form, though this rarely occurs for thin film growth, as eventually, the $r^{4}$ size dependence in Eq. 3 causes the rate of coalescence completion to decrease. This marks the end of the nucleation and growth stage of film formation, as it leads to several substantial changes in the surface morphology.

\subsubsection{MORPHOLOGICAL GROWTH TRANSITIONS}

The $r^{4}$ dependence of the coalescence completion time means that the size of islands eventually becomes prohibitively large for surface diffusion to be an effective means of mass transport. Coalescence eventually ceases altogether, and thin films (both epitaxial systems and growth on amorphous surfaces) are typically seen to go through a percolation ${ }^{5}$ transition, where most islands are interconnected without coalescing and form a web spanning across the entire substrate. [103,109-115] For metals grown on insulating substrates, this transition is easily measurable, as the film begins to conduct electrically for the first time. It is important to note that the occurrence of coalescence and percolation is intimately tied to the intrinsic self-surface diffusivity of the film material. Typically for materials with high melting temperatures such as $\mathrm{Pd}$, coalescence is not seen to occur at all at room temperature and the percolation transition is reached shortly after islands begin to impinge each other. [116-120]

\footnotetext{
${ }^{5}$ This comes from the theory of percolation, which is the study of statistical properties of a spanning cluster of occupied sites on a discrete lattice (site percolation), or a spanning cluster of discs on a continuous surface (continuum percolation), as well as their three-dimensional analogues. A review can be found in [115].
} 
In order to model the occurrence of percolation in thin film growth, the notion of “interrupted" coalescence was proposed by Yu et al, where islands below a critical size would coalesce instantaneously upon impingement and cease to coalesce upon reaching the critical size. [110] This model was later improved by the same authors to include the coalescence dynamics of by Eq. 3 , in what they called the "kinetic freezing" model. [111] In this, it is assumed that the lateral growth, or spread, of islands occurs at a rate

$$
t_{\text {spread }} \approx \alpha r / F \Omega[s],
$$

where $\alpha$ is the height-to-radius ratio of the islands, $r[m]$ is again the island radius, $F(M L /$ $s)^{6}$ is the deposition flux and $\Omega\left[\mathrm{m}^{3}\right]$ again the atomic volume. Note that in this type of treatment, the islands themselves are explicitly modeled as hemispheres, i.e. atomistic processes on the islands are omitted. By equating Eq. 5 to Eq. 3 , the authors calculated the critical island radius for percolation (using $\rho_{A}=\Omega^{-2 / 3}$ ) to

$$
r_{C} \approx\left(\alpha D_{s} \gamma \Omega^{1 / 3} /\left(k_{B} T F\right)\right)^{1 / 3}=(\alpha B / F \Omega)^{1 / 3}
$$

Later, other authors emphasized that this condition was in fact met before the percolation transition, at a stage when the film is predominantly comprised of "elongated" structures, i.e. islands that have partially coalesced. $[109,121,122]$ This transition was thus named the elongation transition, and by assuming a linear relation between the nominal film thickness $\theta[M L]^{7}$ and the average island radius, Eq. 6 was used to represent a scaling relation between $\theta$ at the elongation transition, $B$ and $F$,

$$
\theta_{\text {elong }} \propto(B / F)^{1 / 3} .
$$

The same authors also pointed out that for materials with intrinsically low self-diffusivity, coalescence may not occur at all during film growth and the scaling behavior of the saturation island density $N_{\text {sat }}$ would be reflected in $\theta_{\text {elong }}$, i.e.

$$
\theta_{\text {elong }} \propto(D / F)^{1 / 7}
$$

for $i^{*}=1$ and three-dimensional growth. The saturation island density will be discussed in the next section. Schematic illustrations of possible surface configurations in the different stages of early film formation by island nucleation, growth and coalescence are depicted in Figure 8 .

\footnotetext{
${ }^{6} M L\left[\right.$ atoms $\left./ m^{2}\right]$ refers to "monolayers", i.e. the number of atoms in a single atomic layer of the system lateral size.

${ }^{7}$ This is also often referred to as "material coverage" or simply "coverage", stemming from the theory of twodimensional growth where the amount of material and surface coverage are one and the same.
} 
a)

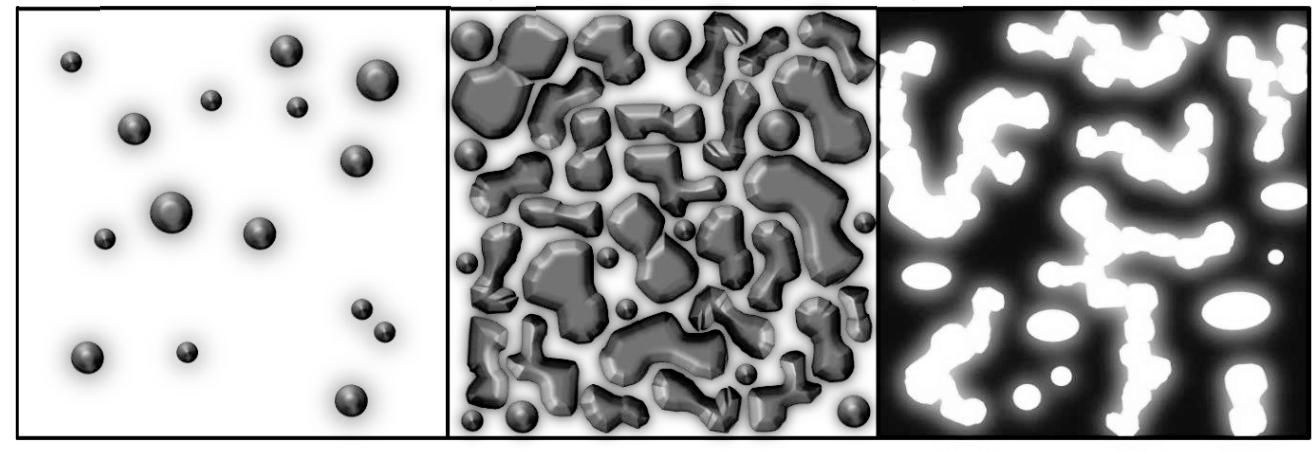

Figure 8. Illustrations of a) the nucleation and growth stage of three-dimensional thin film formation, b) the elongation transition, when the surface is predominantly covered by islands in the process of coalescing that form elongated shapes, c) the percolation transition, where the film consists of a connected network that spans across the entire substrate. In all figures, white represents the substrate.

However, the kinetic freezing model does not account for the formation of boundaries at the contact between coalescing islands. Since the islands are single-crystalline and randomly oriented to each other on an amorphous surface, the formation of a boundary also slows coalescence by impeding surface diffusion. $[9,11]$ For small islands, this boundary is quickly migrated out of a newly coalesced island, but for larger islands, these may become locked in place as the other mechanisms slowing coalescence become relevant (eventually they will make up the grain boundaries in a polycrystalline film). In this sense, it is possible for the elongation transition to occur earlier than predicted by Eq. 7 if grain boundaries form easily for a specific substrate-film combination. [111] This is also indicated in the STM results of reference [79], where grain boundary formation is seen to occur in the late stages of coalescence driven film growth and actively contributes to inducing the percolation transition.

Once percolated, the remaining growth stage to obtaining a continuous film involves filling of trenches and holes in the porous network. As the trenches become narrower, it becomes increasingly difficult to deposit directly into them, and hole-filling must rely on downwards diffusion or funneling of atoms from the top of the film. As the former process is kinetically limited by the Ehrlich-Schwoebel barrier, complete hole-filling is often observed to take an unexpectedly long time; an effect known as the "Zeno" effect. $[79,123]$ Around this time, facets of low-index crystal orientations may become stable on the island surfaces if the temperature is below the surface roughening temperature (not to be confused with kinetic roughening temperature). These facets persist due to the difficulty to nucleate additional 
layers on top of them. $[84,85,89,124]$ Since different low-index surfaces possess different surface diffusivities, the stabilization of facets leads to a competitive growth of certain grains. $[125,126]$ For fcc metals such as Au and Ag, the $\{111\}$ surface typically has a much higher diffusivity than the $\{110\}$ or $\{100\}$. [127-129] This makes a $\{111\}$ surface grow laterally, as atoms tend to diffuse quickly to the edges of the facet and cross the step there, while the other two surfaces will grow more outwards due to the longer residence time of atoms on these surfaces. At very low temperatures (typically below room temperature), this difference in diffusivity is sufficient to cause a height difference to develop between different grains, leading to $\langle 100 / 110\rangle$ out-of-plane oriented grains overgrowing the out-of-plane $<111>$ oriented grains. However at room temperature and above, the differences in diffusivity are less pronounced, leading to the reverse growth behavior. $<100 / 110>$ grains will still grow higher than $<111>$ oriented grains initially, but the increased adatom mobility on $\{100 / 110\}$ facets allows material to be traded onto $\{111\}$ facets. This effect is enough to tip the scale in favor of the $<111>$ oriented grains. Since these are larger in the lateral directions and the height difference between grains is less pronounced than at lower temperatures, $<111>$ oriented grains begin to catch a larger part of the incident flux and slowly outgrow the $<100 / 110>$ grains instead.

As the current work deals with the initial stages of polycrystalline film formation, further microstructural evolution of thin films will not be discussed here; a review can be found in reference [9]. In the next section, mathematical models of the nucleation and growth description given in this and the previous sections are presented.

\subsection{NUCLEATION THEORY}

\subsubsection{Classical treatment}

Very briefly, the classical description of nucleation will be given for sake of comparison to the atomistic treatments in sub-sections 2.2.2 and 2.2.3. Homogeneous nucleation of liquid droplets in a vapor is typically described in textbooks on thermal physics. [18] Under equilibrium conditions, the chemical potential difference $\Delta \mu$ between an infinitely large droplet and the surrounding vapor can be set to $\mu_{g}-\mu_{l}$. If this difference is positive, the liquid is more stable than the vapor, for it has a lower free energy. In reality, the large surfaceto-bulk ratio of small, initially nucleated droplets endows them with large surface free energies, making them unstable as compared to the gas. In order to calculate the rate at which 
stable droplets form, the change in Gibbs free energy due to formation of a spherical droplet of radius $r$ is calculated as

$$
\Delta G(r)=-\left(4 \pi r^{3} / 3\right) n_{l} \Delta \mu+4 \pi r^{2} \gamma[e V]
$$

where $n_{l}$ is the concentration of atoms in the liquid and $\gamma$ is again the isotropic surface free energy. The first term represents the contribution to change from the bulk of the droplet (bulk free energy), while the second term represents the contribution from its surface (surface free energy), with the latter dominating for small droplets, as depicted in Figure 9. However, since the (negative) bulk free energy grows as $r^{3}$, it eventually overpowers the $r^{2}$ contribution form the surface, and a maximum in $\Delta G$ can be found at a critical radius $r_{C}$ with the value $\Delta G_{C}$. By finding the zero in the derivative of Eq. 9 ,

$$
d \Delta G / d r=0=-12 \pi r^{2} n_{l} \Delta \mu+8 \pi r \gamma,
$$

$r_{C}$ can be found as $2 \gamma / 3 n_{l} \Delta \mu$. For $r<r_{C}$, droplets will tend to evaporate randomly to lower the systems total free energy, while for $r>r_{C}$, the total free energy is instead lowered as droplets grow larger. Droplets with $r=r_{C}$ are called critical clusters, since depending on spontaneous thermal fluctuations, these may either become stable or fall apart.

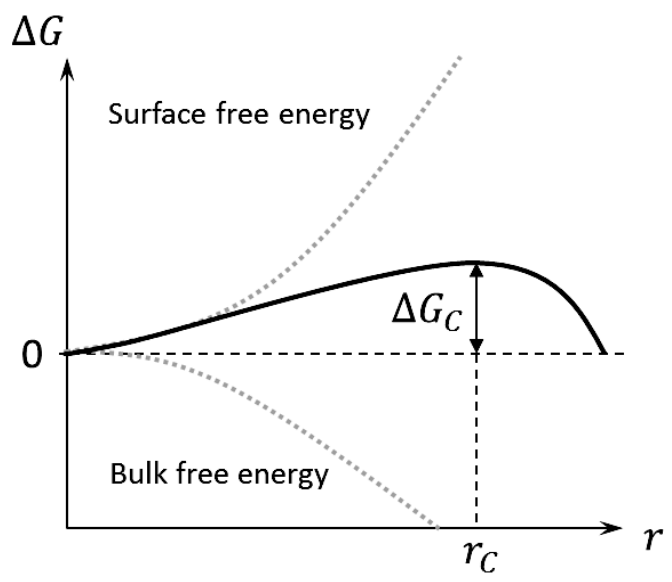

Figure 9. Schematic illustration of the relationship between surface free energy and bulk free energy which combine to yield the Gibbs free energy $\Delta G$ of a (hemi-) spherical particle as functions of the particle radius, $r$. At a critical radius $r_{c}$, a maximum in $\Delta G$ occurs. Particles larger than $r_{c}$ form stable clusters since $\Delta G$ decreases with increasing $r$ while particles smaller than $r_{c}$ will tend to fall apart as $\Delta G$ decreases for decreasing $r$. 


\subsubsection{ATOMISTIC TREATMENT}

For thin film growth, the size of critical clusters is typically on the order of one to a few atoms. At such a small size, it is not possible to define surface and bulk energies; an atomistic nucleation theory is required. A discrete analogy to $r_{C}$ was derived by Walton, called the "critical nucleus size" $i^{*}$ [atoms], where an island with size $i^{*}+1$ is considered stable. From this, a relation that calculates the density of nuclei with this size as a function of the adatom density $N_{1}$ was derived, known as the Walton relation $[7,130]$

$$
N_{i^{*}}=c_{i^{*}} N_{1}^{i^{*}} \exp \left(E_{i^{*}} / k_{B} T\right)\left[1 / m^{2}\right]
$$

where $c_{i}$ reflects the number of optimal configurations of a nucleus with the binding energy $E_{i^{*}}$. Having now a way to describe islands of atomistic proportions, it became possible to reformulate a set of rate equations based on the Smoluchowski coagulation equation, [131] that accounts for the "mean field" kinetics of thin film nucleation in terms of the deposition rate $F$ and substrate diffusivity $D$. This is called a "mean field" treatment because it takes into account the effect of weak long range interactions among objects in a system, which in this case mainly refers to the relation between adatom diffusion on the substrate and their interactions with each other as well as islands. [18] The general rate of change in the adatom density is given by $[77,108,132-137]$

$$
\begin{aligned}
d N_{1} / d t & =F-\left(i^{*}+1\right) D \sigma_{1} N_{1} N_{i^{*}}-D N_{1} \sum_{s \geq i^{*}} \sigma_{s} N_{s} \\
& -\left(i^{*}+1\right) F A_{i^{*}} N_{i^{*}}-F \sum_{s \geq i^{*}} A_{s} N_{s}
\end{aligned}
$$

where $\sigma_{1}$ and $\sigma_{s}$ are known as capture numbers that represent the probability of an adatom and island respectively to catch another adatom, $N_{S}$ is the density of islands of size $s$ [atoms] and $A_{s}$ is the effective capture area of such islands projected onto the substrate. $A_{i^{*}}$ represents the effective capture area of a cluster with the critical size. The second term on the right-handside of Eq. 12 represents the nucleation of stable nuclei, with associated loss of $i^{*}+1$ adatoms, the third term represent the attachment of adatoms to stable nuclei and the fourth and fifth terms represent the loss of adatoms due to nucleation and direct capture from the flux. For sake of brevity, terms representing replenishing of the adatom density due to reversible attachment are not discussed here. [108] The corresponding rate of change in the density of stable islands is given by

$$
\sum_{s \geq i^{*}} d N_{s} / d t=D N_{1}\left(\sigma_{s-1} N_{s-1}-\sigma_{S} N_{S}\right)+F\left(A_{s-1} N_{s-1}-A_{S} N_{S}\right) \quad \text { Eq. } 13
$$

where the two terms in the first parenthesis represent the gain and loss of size $s$ islands due to diffusive capture and the two terms in the second parenthesis represent the same but for direct 
capture from the flux. The contribution of coalescence to the rate equation of stable islands is difficult to include, with some authors attempting to directly implement the Smoluchowski coagulation equation [138]

$$
\sum_{s \geq i^{*}} d N_{s} / d t=\frac{1}{2} \sum_{i+j=s} K_{i j} N_{i} N_{j}-N_{s} \sum_{j=1}^{\infty} K_{s j} N_{j}
$$

where $K_{i j}$ is the impingement rate of two islands of size $i$ and size $j$ that can form an island of size $s$, and $K_{s j}$ is the impingement rate of two islands of size $s$ and $j$ that will reduce the density of size $s$ islands. This is by far the most inclusive representation of coalescence in rate equation form, but the impingement rates $K_{i j}$ and $K_{s j}$ are difficult to describe analytically, and a simpler version of Eq. 14 that was proposed earlier is often used, [77,139,140]

$$
\sum_{s \geq i^{*}} d N_{s} / d t=-C N_{s} d Z_{s} / d t
$$

where $Z_{s}=N_{s} A_{s}$ is the surface coverage and $C$ is a constant that accounts for the effect of ordering among islands. In Eq. 15 , the island impingement rate has simply been described as a function of their areal expansion rate. However, neither Eq. 13 nor Eq. 14 takes into account the dynamic nature of the coalescence of solid particles and assumes this to occur instantaneously upon impingement. Owing to the limited accuracy and thus applicability of rate equation treatments of coalescence, such effects are rarely included when thin film nucleation is characterized mathematically. Thus, rate equation theory is typically used to describe "pre-coalescence" growth stages, with theories like kinetic freezing taking over at later stages.

A central prediction of the atomistic nucleation theory is the scaling behavior of the saturation island density $N_{\text {sat }}$, occurring when the atom capture rate at islands exceeds the nucleation rate. To calculate this, simplified versions of Eq. 12 and Eq. 13 are often used, [7]

$$
\begin{array}{cc}
d N_{1} / d t=F-2 D \sigma_{1} N_{1} N_{i^{*}}-D \sigma_{x} N N_{1} & \text { Eq. } 16 \\
d N / d t=D \sigma_{1} N_{1} N_{i^{*}} & \text { Eq. } 17
\end{array}
$$

where all stable islands are assumed to behave in the same way and collectively represented by the density $N$ and average capture number $\sigma_{x}, i^{*}=1$ is assumed (i.e. dimers are considered stable) and direct capture from the flux is considered negligible to the small scale of islands in the initial nucleation stages. Beginning from an empty surface, the adatom density increases roughly as Ft (nucleation is negligible at this point, see Figure 10), in a transient growth regime. As nucleation begins to occur significantly, the adatom density begins to decrease. Capture at existing islands begins to compete with nucleation for adatoms, 


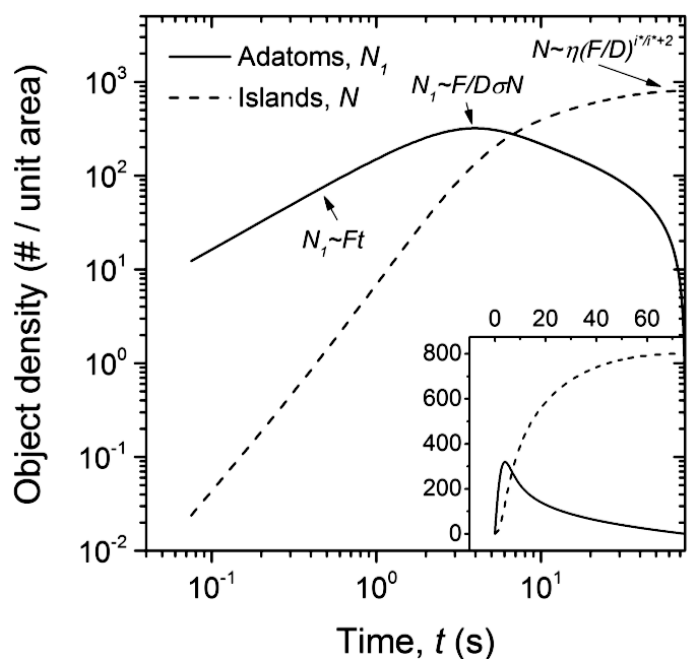

Figure 10. Log-log graph of the density of adatoms and islands from numerically integrated rate equations. Key values of the adatom and island densities based on rate equation analysis are indicated. The inset shows the same data in linear scale to give a sense of the difference between adatom and island density evolutions both in time and magnitude.

and eventually the adatom density reaches a maximum while nucleation begins to decrease as well. By setting Eq. 16 equal to zero under the assumption that nucleation is negligible, an analytical solution to the adatom density can be found,

$$
N_{1}=F / D \sigma_{x} N\left[1 / m^{2}\right]
$$

Integrating Eq. 17 with Eq. 16 for $N_{1}$ and Eq. 11 for $N_{i^{*}}$ produces the expression

$$
N=\eta(F / D)^{i^{*} /\left(i^{*}+2\right)} \exp \left(E_{i^{*}} / k_{B} T\right)\left[1 / m^{2}\right]
$$

where $\eta=\left(\left(i^{*}+2\right) \theta c_{i^{*}} \sigma_{1} / \sigma_{x}^{i+1}\right)^{1 /\left(i^{*}+2\right)}$. Eq. 19 calculates the value that the island density approaches as the nucleation rate eventually disappears, and can thus be used to represent the scaling behavior of the saturation island density: $N_{s a t} \propto(F / D)^{1 / 3}$ for $i^{*}=1$ and $E_{i^{*}}=0$. The linear dependence of the $\eta$-parameter on material coverage $\theta$ means Eq. 19 is strictly valid for two-dimensional growth, where surface coverage and material coverage are synonymous. In order to find the scaling behavior of $N_{\text {sat }}$ for three-dimensional islands $\eta$ must be related to the actual surface coverage $\phi$ through the substitution

$$
\phi \sim N S^{2 / 3} \sim N(\theta / N)^{2 / 3} \sim N^{1 / 3} \theta^{2 / 3}
$$

where $S=\theta / N$ is the average island size. [77,108] Using Eq. 20 in Eq. 19 , one finds the scaling relation 


$$
N \propto \phi^{3 /\left(2 i^{*}+5\right)}(F / D)^{2 i^{*} /\left(2 i^{*}+5\right)}
$$

For $i^{*}=1, N_{\text {sat }} \propto(F / D)^{1 / 7}$ is recovered.

For more quantitative analysis of three-dimensional growth, $\eta=0.25$ is often used as an approximation based on kinetic Monte Carlo simulation results. [8,137] This serves to exemplify the difficulty in analytically calculating $\eta$ and in particular the capture numbers $\sigma_{1}$ and $\sigma_{s}$ or $\sigma_{x}$. Much work has gone into finding an analytical solution to these by including the effects of island size, shape and the adatom diffusion profile on the substrate around islands. [141-143] The detailed study of capture numbers lies far beyond the scope of this simple introduction to nucleation theory and will not be given here.

\subsection{FILM GROWTH UNDER PULSED VAPOR FLUXES}

The atomistic models of film nucleation and growth described in section 2.1.1 and 2.1.2 should also hold for pulsed vapor fluxes, with reservation for energetic bombardment effects. $[15,16,144]$ However, nucleation in pulsed deposition, as opposed to continuous deposition which has been assumed so far, is not as easily analyzed in the framework of rate equations as integration cannot be performed when the flux $F$ is discontinuous. [145] Instead, the general scaling behaviors of $N_{\text {sat }}$ given by Eq. 19 and Eq. 21 were adapted to pulsed deposition by comparing the relative time-scales of deposition and substrate diffusion. $[5,12,13]$

A pulsed vapor flux can be characterized by the duration of the pulse or pulse on-time $t_{\text {on }}[s]$, the duration of a period $1 / f[s]$ where $f[\mathrm{~Hz}]$ is the frequency and the instantaneous deposition rate $F_{i}[M L / s]$ (see Figure 11). For typical pulsed deposition techniques, $t_{\text {on }} \ll$ $1 / f$ may be assumed. The total amount deposited per pulse $F_{P}[M L / p u l s e]$ is then the product $F_{i} t_{o n}$, and the average deposition rate $F_{a v}=f F_{P}[M L / s]$. The two time-scales $1 / f$ and $t_{o n}$ can be directly compared to the adatom, or adatom lifetime $\tau_{m}=1 / N_{s a t} D[s]$, which represents the duration the adatom density persists on the substrate once the pulse is turned off. The disappearance of the adatom density is again due to nucleation or adatom capture at islands. Hence, the saturation island density is used to represent the maximum loss of adatom which is in return the cause of island density saturation. In terms of nucleation, three different scaling behaviors of $N_{\text {sat }}$ can be identified for pulsed vapor deposition for decreasing diffusivity $D$ or increasing adatom lifetime $\tau_{m}$. If $\tau_{m} \ll t_{o n}$, the adatom density vanishes as soon as the pulse is turned off, effectively allowing nucleation only during the pulse. The 
a)

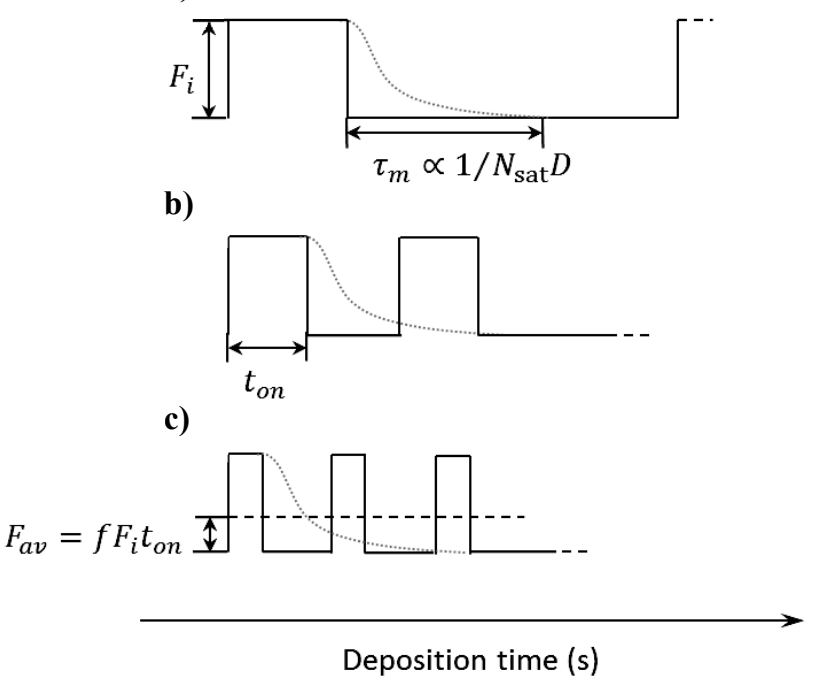

Figure 11. Schematic illustration of the relationship between deposition time-scales and the adatom lifetime for pulsed deposition in three different pulse configurations. These correspond to the a) high diffusion regime, where adatoms vanish rapidly once the pulse is turned off, b) the intermediate diffusion regime, where adatoms persist for the entire duration of the pulse off-time and c) the low diffusion regime, where adatoms persist over many successive pulses. The different pulse parameters described in the text are also indicated.

deposition rate $F$ in Eq. 19 and Eq. 21 can then be replaced by $F_{i}$, and a scaling relation $N_{\text {sat }} \propto\left(F_{i} / D\right)^{\chi}$ is recovered, with $\chi$ being either $i^{*} /\left(i^{*}+2\right)$ for two-dimensional growth or $2 i^{*} /\left(2 i^{*}+5\right)$ for three-dimensional growth. The remaining two nucleation scaling behaviors will be described in terms of $\chi$ with the understanding that it is different for the twodimensional and three-dimensional cases. If $t_{o n} \ll \tau_{m}<1 / f$, adatoms persist into the time between pulses and contribute to nucleation even in the absence of deposition. This gives rise to a unique scaling behavior that is solely dependent on the amount deposited per pulse, $N_{\text {sat }} \propto F_{P}^{\chi / 1+\chi}$, calculated by integrating Eq. 17 assuming an exponential adatom decay rate. [13] Finally, if the adatom density persists across multiple pulses, $t_{o n} \ll 1 / f \ll \tau_{m}$, nucleation may essentially occur at any time, and the substrate effectively "sees" only the average deposition rate $F_{a v}$. This gives rise to a scaling behavior identical to the continuous deposition case, $N_{s a t} \propto\left(F_{a v} / D\right)^{\chi}$ if $F_{a v}$ is made comparable to a continuous deposition rate $F$. The conditions for these scaling relations were later re-described in terms of the deposition 
rates $F_{i}, F_{P}$ and $F_{a v}$, [5]; results of the two different approaches as well as the different scaling behaviors of $N_{\text {sat }}$ are summarized in Table 1.

As mentioned initially, the atomistic processes involved in nucleation and growth under a pulsed deposition are likely similar to the continuous deposition case. However, the behavior of coalescence and occurrence of morphological transitions (elongation and percolation) may be altered drastically. It has been reported that the energetic bombardment typically associated with pulsed deposition may cause the creation of additional surface adatoms on coalescing islands, effectively promoting coalescence even for large island sizes. [14,146] This tends to delay the onset of elongation and percolation, such that the nominal film thickness becomes higher than that predicted by Eq. 7 .

Table 1. Summary of the three different nucleation regimes found in pulsed vapor deposition both in terms of time-scale relations (first column) as well as rate relations (second column). The scaling function of $N_{\text {sat }}$ is given in colum three.

\begin{tabular}{cccc}
\hline $\begin{array}{c}\text { Nucleation } \\
\text { regime }\end{array}$ & $\begin{array}{c}\text { Time-scale } \\
\text { relation }\end{array}$ & Access criteria & $\boldsymbol{N}_{\text {sat }} \propto$ \\
\hline High diffusion & $\tau_{m} \ll t_{\text {on }} \ll 1 / f$ & $\left(F_{i} / D\right)^{1-\chi}<F_{P}$ & $\left(F_{i} / D\right)^{\chi}$ \\
$\begin{array}{c}\text { Intermediate } \\
\text { diffusion }\end{array}$ & $t_{\text {on }} \ll \tau_{m}<1 / f$ & $\left(F_{a v} / D\right)^{1-\chi}<F_{P}<\left(F_{i} / D\right)^{1-\chi}$ & $F_{P}^{\chi /(1-\chi)}$ \\
Low diffusion & $t_{\text {on }} \ll 1 / f \ll \tau_{m}$ & $F_{P}<\left(F_{a v} / D\right)^{1-\chi}$ & $\left(F_{a v} / D\right)^{\chi}$ \\
\hline
\end{tabular}




\section{Kinetic Monte Carlo simulations}

\subsection{Physical MOdel}

For an initial venture into simulations of film growth using kinetic Monte Carlo, many of the atomistic processes described in Chapter 2 are omitted, generalized or averaged over larger length-scales. This is often known as coarse-graining, and helps to speed up the computational effort as well as putting focus on a few, key atomic processes.

The kMC simulations used in the appended articles is largely based on the work of Warrender from reference [147], which in turn is based on the simulations of Carrey and Maurice [109,121] but adapted to include the pulsed deposition flux described in section 2.3. In these and the current model, a simple cubic lattice is used with a lattice spacing that is equal to $a$, the lattice spacing of the film material. Deposition occurs by randomly selecting a site on the substrate and placing a new atom, i.e. transport in the vapor phase is not modeled, energetic effects are ignored and the sticking coefficient is unity everywhere on the substrate and growing film. Only the basic substrate diffusion given by Eq. 1 is used to model atomic motion, while islands are represented by hemispheres with a given center-of-mass and radius in analogy to a "point island model". [29,103,134,142] This enables a concentrated study of the competition between the deposition rate (or rates in the case of pulsed deposition) and the substrate diffusivity, which are the main parameters governing the nucleation behavior, and also speeds up the computational effort.

Dimer nucleation is considered irreversible, i.e. $i^{*}=1$ and the smallest islands consists of two atoms. Islands may grow by single adatom attachment at the edge or by direct deposition onto the island, whereby the adatom is placed at the closest position on the island that preserves a hemispherical shape. The shape-preservation is justified by considering a film material with efficient edge diffusion and corner crossing on and around the islands, which facilitates shape equilibration as per the descriptions of section 2.1. As adatoms may come from all directions, this tends to shift the center-of-mass of the island over short times (up to a few attachments) but the time-averaged center-of-mass is preserved. The island radius is calculated by a simple volume-conserving geometrical relation

$$
R=(3 s \Omega / 2 \pi)^{1 / 3}[m]
$$


where $s$ is the island size in atoms and $\Omega$ the atomic volume, and this is recalculated for each atom added to the island.

Island-island interactions are modeled based on the kinetic freezing model and thus Eq. 3 is used to calculate the coalescence completion time. Coalescence events are considered between pairs of islands at a time (see Figure 12), and mass transport between the islands is not strictly modeled. The latter makes the coalescence treatment a "binary" (start-stop) process. This means that a timer is started upon impingement between two islands with a limit set by Eq. 3 that counts down with the simulation time. During coalescence, the islands are allowed to grow independently from each other, with the timer limit being adjusted with the size of the smallest island in the pair. Upon reaching the timer limit, the two island are immediately merged into a single new island at their averaged center-of-mass and with their combined sizes. The dynamic dependence of the timer limit (coalescence completion time) with island size is in line with the notion that island coalescence is driven by deposition (as discussed in section 2.1.3) and allows for a study of the competition between coalescence and island growth. Faceting of the islands is not considered a dominating effect at the small length-scales associated with growth up to the elongation transition.

To track the onset of the elongation transition, impinging islands are collected into "clusters", as the one depicted in Figure 12. Specifically, a lone island is considered a cluster of size one, while the cluster size is not limited upwards. In this way, when the average cluster size reaches two, the surface is predominantly covered by islands that have impinged other islands. Due to the relatively small surface areas studied with the simulations, the effects of secondary nucleation become amplified as these islands will be of comparable size to the primarily nucleated islands. This tends to skew the onset of the elongation transition such that the scaling behavior of $\theta_{\text {elong }}$ deviates from Eq. 7 . As such, secondarily nucleated islands are

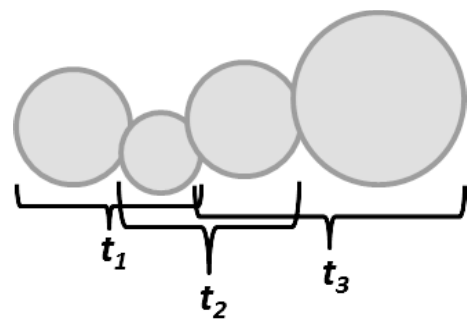

Figure 12. Illustration of a coalescing cluster of islands in the simulation, with independent coalescence timers $t_{1}, t_{2}$ and $t_{3}$ between each impinging pair of islands. 
allowed to form, but are not counted during the calculation of simulation statistics such as the average island or cluster size.

\subsection{SimULATION ALGORITHM}

A flow chart of the entire simulation algorithm is given in Figure 13, and is similar to typical lattice-based kMC algorithms found in the literature. [74,148-150] The $-v s$ flag is used to produce visualization output of either atoms or islands (described later).

Once input parameters such as deposition rate, diffusion barrier and coalescence parameters have been read, the algorithm proceeds to choose between a deposition or diffusion event, where the deposition probability is based on the deposition rate, and the diffusion probability is based on the product between diffusivity and adatom density. If deposition is chosen, an atom is placed on the substrate per the description in the previous section and indexed into a list of mobile adatoms. If diffusion is chosen, and an adatom is selected randomly from this list, its direction is also chosen randomly (N, S, E or W on a simple cubic lattice) and the atom is moved. If the final position of the atom, irrespective of whether it was deposited or diffused, neighbors another adatom, an island is nucleated out of these and is indexed into a list of islands as well as clusters (single islands count as a cluster of size one). If the final position of the atom is at the edge of an island, the atom becomes instantaneously incorporated into the island per the description in the previous section. If island attachment occurred, it is checked whether the growth of the island led to successive attachments of nearby adatoms to avoid unphysical behavior. This is done recursively until no further atoms can be incorporated into the island, and concludes the atomistic processes treated in a simulation step.

The next function involves checking for impingement between the island that grew and its neighbors. For each impingement event, a coalescence completion timer is started for the two associated islands. In other words, the island that grew may impinge (and thus coalesce) with multiple islands simultaneously, with an independent timer for each impinging neighbor. Once this is done, the next function proceeds to check for coalescence completion, which occurs by replacing the two previous islands with a new one as per the description in the previous section (Figure 14a). However, this function becomes rather involved as multiple special cases may occur when coalescence completes within a multi-island cluster, as illustrated in Figure 14b-d. Beginning with the simplest case of coalescence completion in a cluster of two islands, new impingement events are immediately checked upon creation of the 


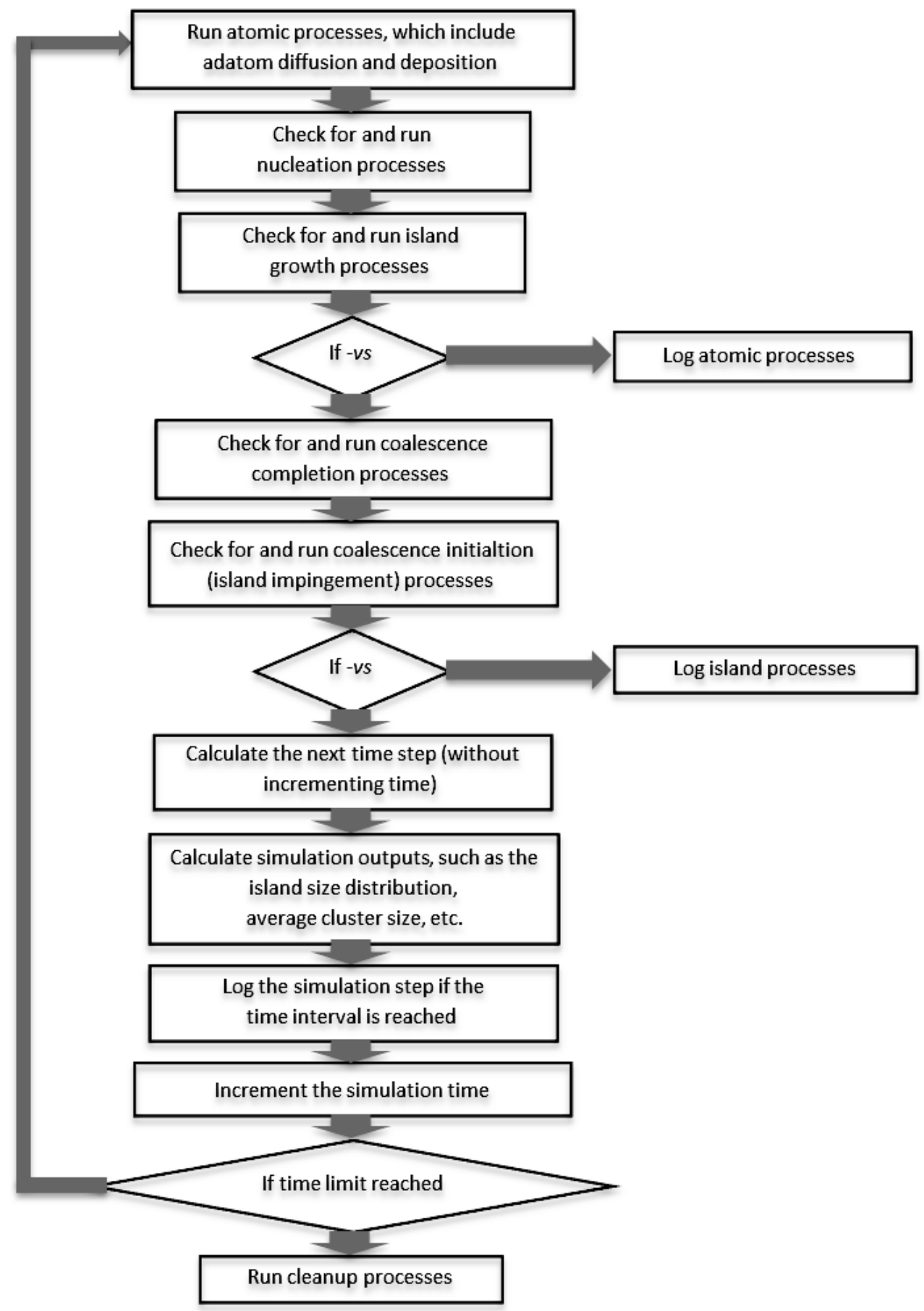

Figure 13. Flow chart of the simulation algorithm. 
a)

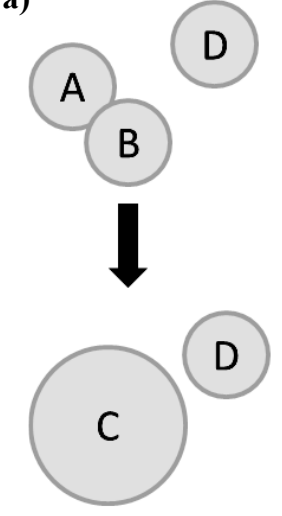

b)

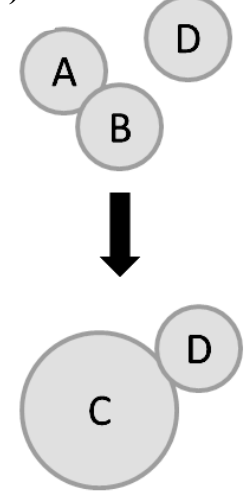

c)

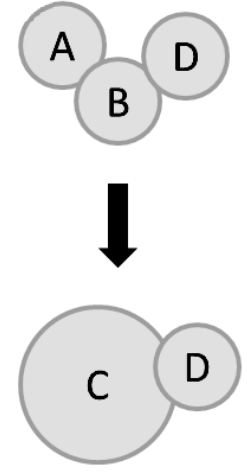

d)

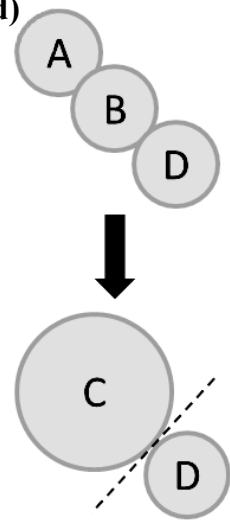

Figure 14. Illustrations of different coalescence completion scenarios that occur during a simulation. Islands $\mathrm{A}$ and $\mathrm{B}$ coalesce into $\mathrm{C}$ a) without affecting $\mathrm{D}$, b) causing impingement between $\mathrm{C}$ and $\mathrm{D}$, c) transferring the previous coalescence timer between $\mathrm{B}$ and $\mathrm{D}$ to $\mathrm{C}$ and $\mathrm{D}$ and d) causing $\mathrm{D}$ that was originally connected to B to seem visually disconnected from $\mathrm{C}$.

merged island, along with the associated creation of new coalescence timers (Figure 14b). For larger clusters (Figure 14c), if the newly coalesced island (C) is still connected to any islands in the original cluster (D), the original coalescence timer is transferred to the new island (C). This is to account for the amount of coalescence progression that already occurred between the original islands (B and D) since coalescence does not have to start over from the beginning simply because the local environment of an island changed. Finally in Figure 14d, this transfer of a coalescence timer is also done if an island (D) in the original cluster becomes visually separated from the newly coalesced island (C), as it is assumed that the necks formed between islands in a cluster are not broken upon a change in the local environment either. The visual separation is a drawback of the physical model, since mass transport is not explicitly simulated during coalescence and the islands are strictly hemispherical.

After sorting out the impingement and coalescence relations, the affected clusters are updated by creating new ones from new impingements cause by growth or coalescence completions, removing clusters that underwent coalescence completion and linking multiple clusters that became connected due to impingent.

Once all island-island processes have been dealt with, the step time is calculated. Typically, this is calculated as 


$$
t_{\text {step }}=1 /\left(h_{\text {tot }}+F\right)[s]
$$

where $h_{t o t}=h * \sum N_{1}$ for continuous deposition and the same relation but with $F_{i}$ instead of $F$ for the pulsed case. However, for the pulsed case, the time interval to the next pulse-on or pulse-off may be shorter than the time calculated by Eq. 23 . Likewise in both pulsed and continuous deposition cases, the time interval to a coalescence completion event may also be shorter than Eq. 23 . In all such cases, the shortest time is chosen instead as the time-step and the diffusion and deposition probabilities are scaled down to reflect this shortened time step.

At this point, all basic simulation quantities have been established for the current simulation step, and calculations of average and other desired metrics are carried out. The relevant data, summarized in Figure 15, is written to a log file and the simulation limits are checked. If they are reached, finalization of the simulation is carried out. Otherwise, the simulation proceeds to
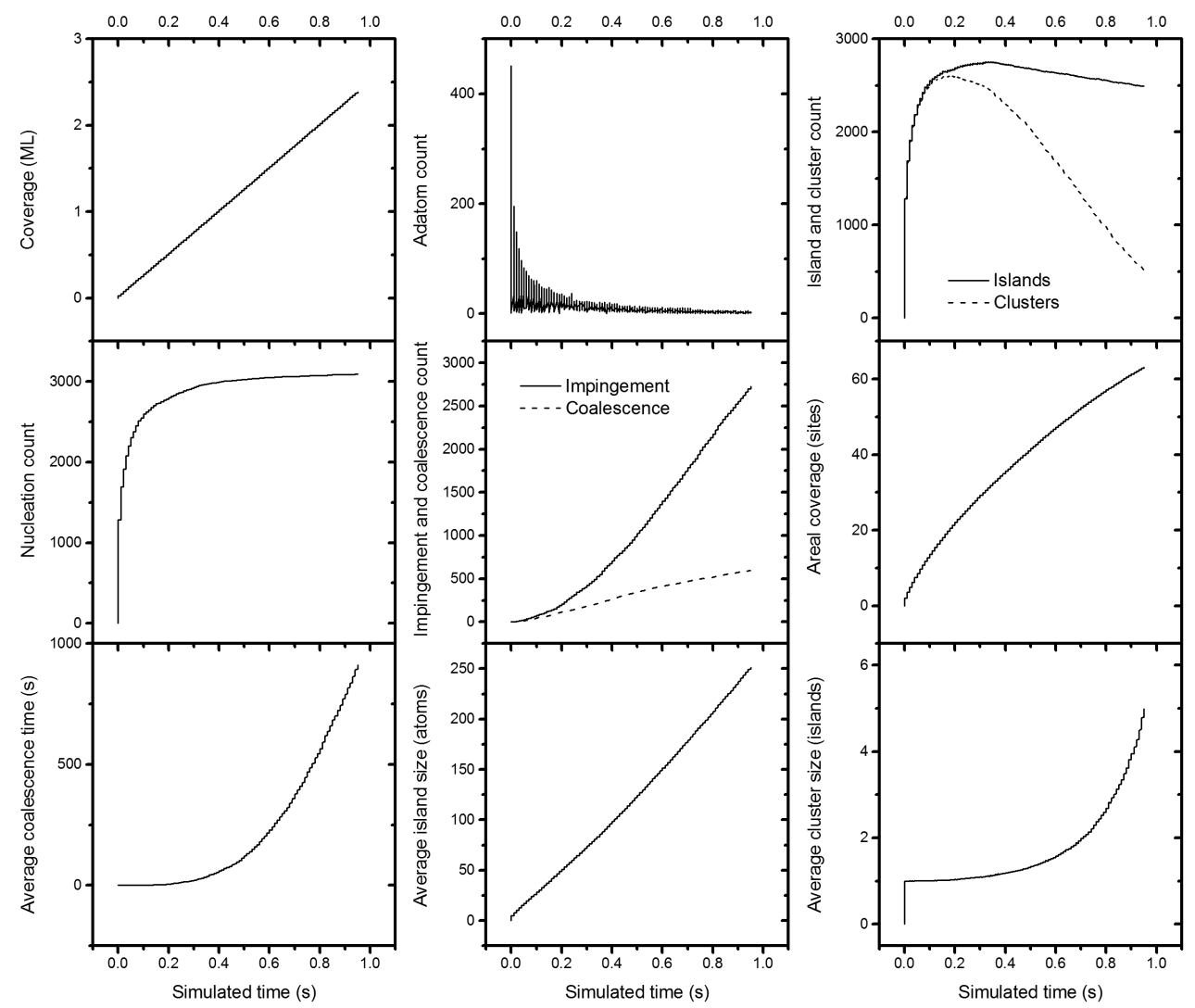

Figure 15. Graphs of the different quantities that can be extracted from the simulations. 
the next step which begins by selecting between deposition and diffusion processes again.

Both continuous and pulsed depositions can be simulated, with the deposition rate for the continuous case calculated as the average deposition rate of an otherwise equivalent pulsed deposition. For regular deposition simulations, three different time limits can be set: a total number of simulation steps, a total length of simulation time or the occurrence of the elongation transition. For the last limit, the simulation will actually progress further along, until the average cluster size reaches five. This is useful in the event the elongation transition is not well defined (e.g. if the average cluster size fluctuates around two) and provides a way to extract results by post-processing the simulation data.

Visualization data can also be produced, though the amount of data becomes unmanageable for larger and/or longer simulations. Two versions of visualization are possible, where one contains a full representation of the simulation, with both atomic motion and island evolution, while the other contains only information on island evolution (see Figure 16). Commonly in molecular dynamics simulations, the position of all objects in the simulation is recorded at set intervals of either simulation time or step, with the total number of objects (typically atoms)
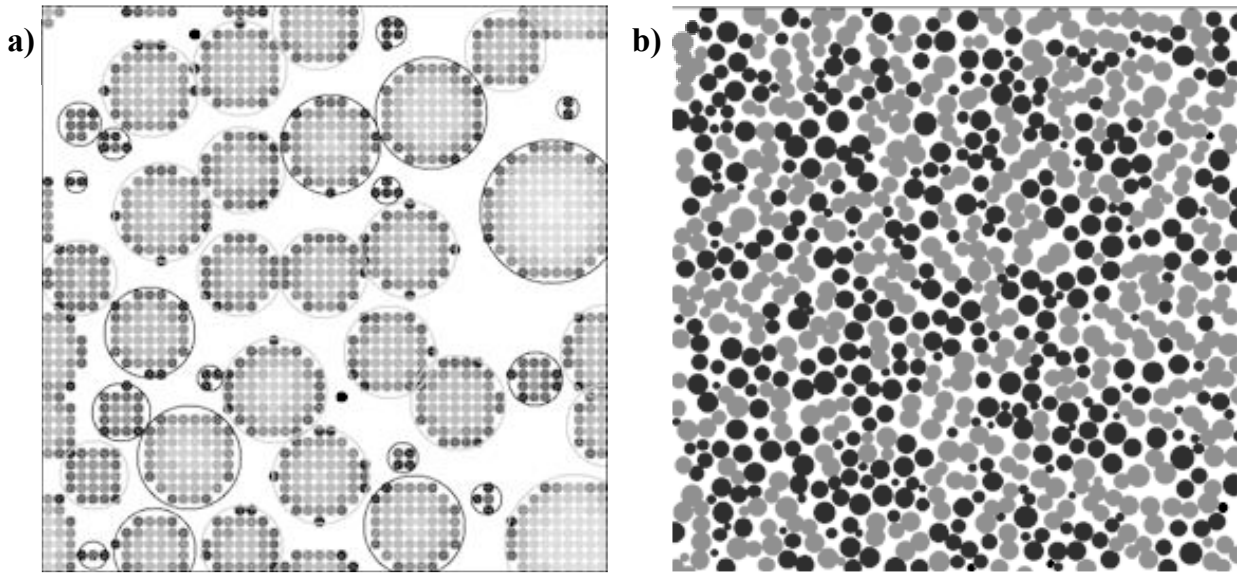

Figure 16. a) Atomistic visualization output, with the filled circles representing atoms, graded in greyscale by height from dark to light. Atoms belonging to islands are encircled by single hollow circles. Dark hollow circles are not in contact with other islands while lighter ones indicate that the island is part of a cluster. b) Island visualization output, again with darker filled circles representing isolated islands while lighter filled circles represent islands in a cluster. Also, secondarily nucleated islands are represented by black filled circles. 
being constant throughout the simulation. In KMC simulations, the number of objects is always changing and can often reach several millions, making it impractical to track everything at every output. Instead, only that which has changed is recorded in each step (or interval of steps), which helps to cut down on the file size of visualization output.

\subsection{MODEL AND SIMULATION ALGORITHM VALIDATION}

\subsubsection{SingLe PULSE EVOLUTION}

By studying the single pulse evolution of island and adatom densities, a number of processes can be validated. These include the rate and uniformity of deposition, the diffusion rate of adatoms as they nucleate and get incorporated into islands, the nucleation rate of islands and also the part of the time step calculation related to deposition and diffusion. Figure 17 shows the island and adatom density evolutions from a single pulse over a period of three seconds from this work. This simulation was run under nearly the same conditions as in reference [151], and reproduces the same maximum island and adatom densities. However, the rate at which the maximum island density is reached is faster than the reference. This is likely due to the use of different simulation box sizes; the reference used a box of $1024 \times$ $1024 a^{2}$ while this work used $768 \times 768 a^{2}$ for the sake of a shorter computation time. The smaller box size makes it easier for adatoms to find each other, thus allowing nucleation

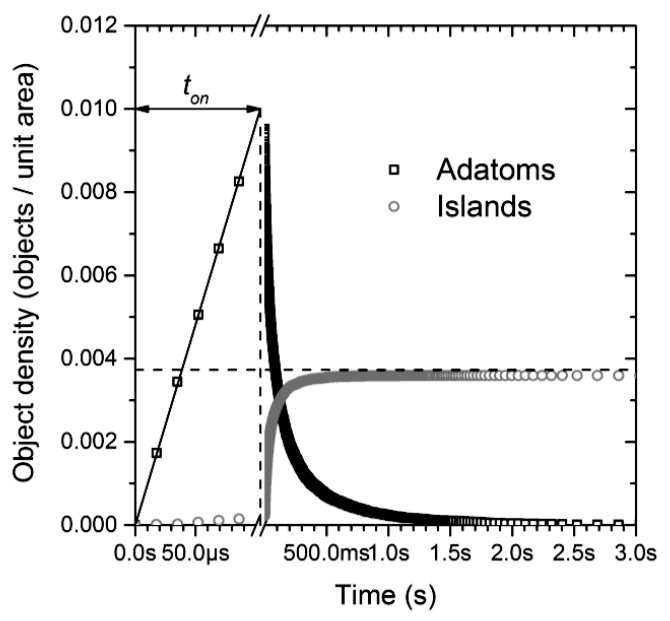

Figure 17. Island and monomer (adatom) density evolution from a single pulse from this work. The pulse width was $100 \mu \mathrm{s}$ and the amount deposited per pulse was $0.025 \mathrm{ML} /$ pulse while $D \approx$ $100 a^{2} / s$. 
events to occur seemingly more rapidly. However, since the amount deposited per pulse is given in terms of a fraction of the box size (equivalently, a fraction of a monolayer) and the same fraction $(0.025 \mathrm{ML} /$ pulse $)$ was used in both cases, there is no discernable difference in the final adatom and island densities between the two works.

It should be noted that the attempt frequencies $v_{0}$ also differed between this work and reference [151]. For this parameter, reference [151] used a value of $5 \times 10^{12} s^{-1}$, while we used $10^{13} s^{-1}$. According to the atomistic nucleation theory, our slightly higher $v_{0}$ should enhance diffusion and promote island growth in favor of nucleation. Thus, assuming that this difference is significant, a higher $v_{0}$ should produce a slower nucleation rate and cannot explain the differences seen in Figure 17. Generally, there is no agreement on the value of $v_{0}$ in the litterature, ${ }^{8}$ with different scientific disciplines using slightly different values. Since reference [151] is a substantial reference to this work, the value of $v_{0}$ was changed to facilitate subsequent validation comparisons and consequently, all simulation results presented in section 3.3.2 and 3.3.3 and the appended papers used a $v_{0}$ value of $5 \times 10^{12} \mathrm{~s}^{-1}$. An interesting detail one might notice in comparing the two panels in Figure 17 is the way data points are distributed along the x-axis. In a), these are bunched up around the end of the deposition pulse while in $b$ ), these are more uniformly distributed. The reason the data points are bunched up in a) is because this work took data points at equal intervals of simulation steps instead of equal time steps. This has no bearing on the results produced by the code in the present work, but merely represents a way to differentiate between the two codes.

\subsubsection{ISLAND DENSITY AND AVERAGE SIZE EVOLUTION IN DROPLET GROWTH}

As was mentioned in section 2.1.3, many analytical results from the study of water vapor condensation can be applied to metal-on-insulator film growth, although certain approximations must be made first. The notion of "droplet growth" in film formation pertains to a case where atomic diffusion on the substrate is completely neglected, and coalescence events are completed instantaneously instead of over a finite period of time. As per the discussions in section 2.1.3 and 2.1.4, this is not very far from the truth when islands are small, but in most material systems as islands grow sufficiently large, droplet growth can no longer describe film growth to an adequate degree of accuracy. Instead, droplet growth simulations can be used as a reasonable way to validate the island impingement characteristics in a simulated growth experiment.

\footnotetext{
${ }^{8}$ For a more in-depth discussion about the attempt frequency, see reference [5].
} 
From the work of reference [139], an analytical expression was formulated that describes island loss mechanics during droplet growth as an exponential decay with material coverage $\theta$

$$
N=N_{0} \exp \left(-A \theta^{2 / 3}\right)
$$

where $N_{0}$ is an initial island density and $A$ is a fitting parameter. In the original work, nucleation was neglected and the study assumed a fixed number of initial islands (corresponding to a density $N_{0}$ ) which were allowed to grow and coalesce instantaneously upon impingement. In order to produce a fair comparison with the simulation results from this work, which do include nucleation, Eq. 24 can only be fitted to the decaying portion of the simulated island density, which begins from $N_{\text {sat }}$, as shown in Figure 18. It can be seen that the simulation data produced from this work is very well fitted by the analytical expression, and this is taken as an indication that the areal distribution of islands, which directly influences the amount of island impingement, evolves correctly. Indirectly, this also confirms that island growth (by both atomic incorporation and coalescence) perform in a correct way.

As a second example of droplet growth, simulation results from the present work have also been compared against reference [109]. In this reference, a simpler version of island loss mechanics was used, but instead an additional analytical expression was provided to describe

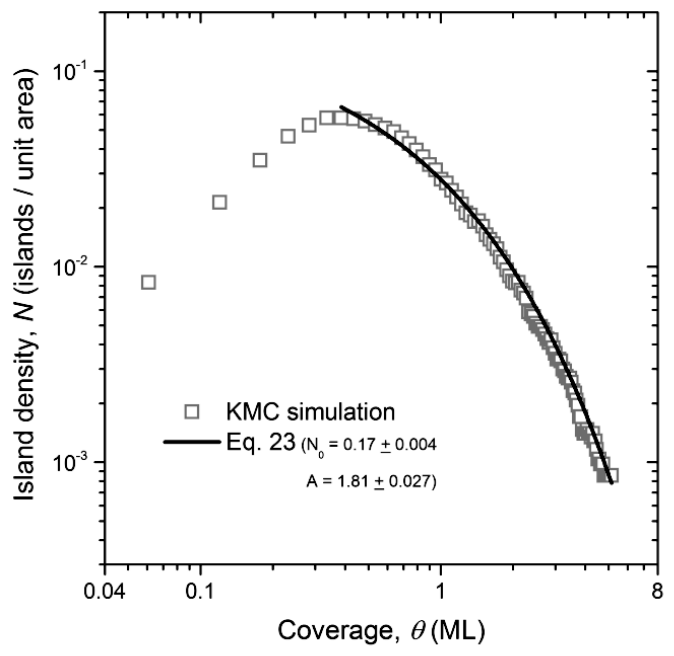

Figure 18. Simulated island density decay during droplet growth, fitted with Eq. 24 . The droplet growth was reproduced by using $D=2 \times 10^{-5} a^{2} / s$ and $B=10^{6} a^{4} / s$, using a continuous deposition rate of $1 \mathrm{ML} / \mathrm{s}$ and simulation box size $128 \times 128 \mathrm{a}^{2}$. 
the evolution of the average island size. The decaying part of the island density $N$ is now described by a simple power-law function,

$$
N=A\left(\theta-\theta_{0}\right)^{-d /(D-d)}
$$

where $\theta_{0}$ represents a shift of the material coverage to exclude the nucleation stage, $d$ and $D$ are the dimensionalities of the substrate and islands respectively and $A$ is a fitting parameter. For $d=2$ and $D=3$, the exponent in Eq. 25 becomes -2 . The average island size $S$ is described by a similar power-law function of $\theta$,

$$
S=B\left(\theta-\theta_{0}\right)^{D /(D-d)}
$$

where $B$ is again a fitting parameter. For $d=2$ and $D=3$, the exponent in Eq. 26 becomes 3. According to literature, the island decay is not as well described by this simpler expression, which can be seen in Figure 19a), where Eq. 25 has been fitted to the same simulated island density as in Figure 18, with the exponent fixed to -2, and compared to the fit of Eq. 24 . Similar results have also been shown in reference [147]. Figure 19b) shows the average island size extracted from the same simulations fitted with Eq. 26 with the exponent fixed to 3. The fit is very poor in the beginning since the growth of islands in the simulations is dominated by atomic incorporation as opposed to coalescence, but the simulated and analytical expressions

a)

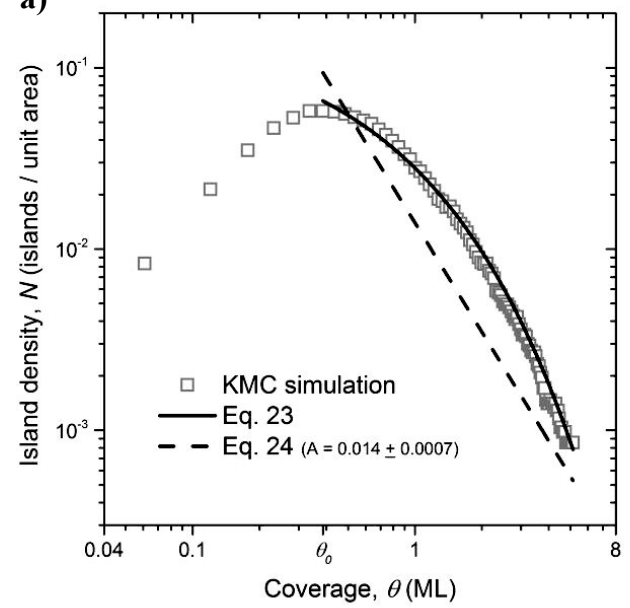

b)

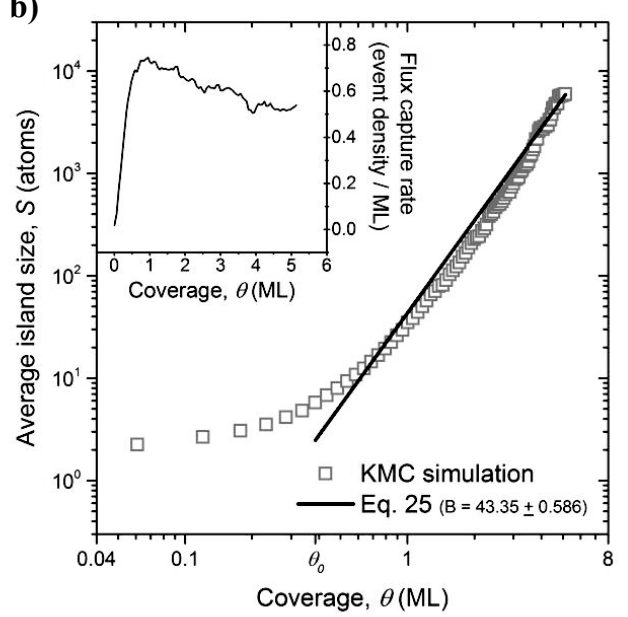

Figure 19. a) Comparison of Eq. 23 and Eq. 24 with respect to how well they fit to simulated island densities from this work. b) The average island size evolution from the same simulation as in a), fitted with Eq. 25. The inset shows the rate of direct capture of material from the flux on top of islands as a function of $\theta$. 
do converge at higher coverages $(\theta>1 M L)$. In this region, the fit between the two is very good, proving that island growth by the combination of atomic incorporation and, most importantly, coalescence performs in a correct manner.

Since there is no atomic diffusion in droplet growth, any island growth by atomic incorporation must necessarily come from the deposition flux, and so the capture rate of atoms from the flux on top of islands is plotted in the inset of Figure 19b). From this plot it can be seen that, after the initial rise, the flux capture rate converges towards a constant value, meaning that if island growth were only dependent on deposition, the average island size would increase linearly with $\theta$ (exponent one in Eq. 26 ). The fact that it does not occur gives insights into the way coalescence influences the average island size, which can be useful when exploring different alternative expressions that separate the effects of different growth mechanisms on the average island size for the purpose of formulating analytical relations.

\subsubsection{TREATMENT OF SECONDARILY NUCLEATED ISLANDS}

Since the secondarily nucleated islands described in section 2.1.3 are systematically excluded in the simulations, it is important to know how such exclusion affects the results. To get a feel for the extent these unwanted objects are present in the simulation, Figure 20 shows the number of primarily and secondarily nucleated islands of sizes two (dimers) and three (trimers) atoms at the elongation transition in Figure 20a) and b), for pulsed deposition at 10 $\mathrm{Hz}$ and $100 \mathrm{~Hz}$ respectively. The graphs clearly show that the proportion of secondarily nucleated islands grows with decreasing deposition frequency. This is due to the increase amount of coalescence (and thus increased amount of re-exposed surface) allowed by the lower average deposition rate at lower frequencies. Less noticeable is that the absolute number of secondarily nucleated islands has also increased marginally from $100 \mathrm{~Hz}$ down to $10 \mathrm{~Hz}$. In Figure 20c) and d), plots of the total island density, including secondarily nucleated islands, is compared to the density of only the primarily nucleated islands. The fact that the curves deviate from each other indicates that secondarily nucleated islands are indeed excluded in a seemingly correct amount from calculations in the simulation. Further investigations into the precise amount of secondarily nucleated islands (beyond trimers) cut from the simulations was not carried out as the simulations were repeatedly able to reproduce the scaling behavior of Eq. 6 at low deposition frequencies where coalescence completion occurs frequently. 


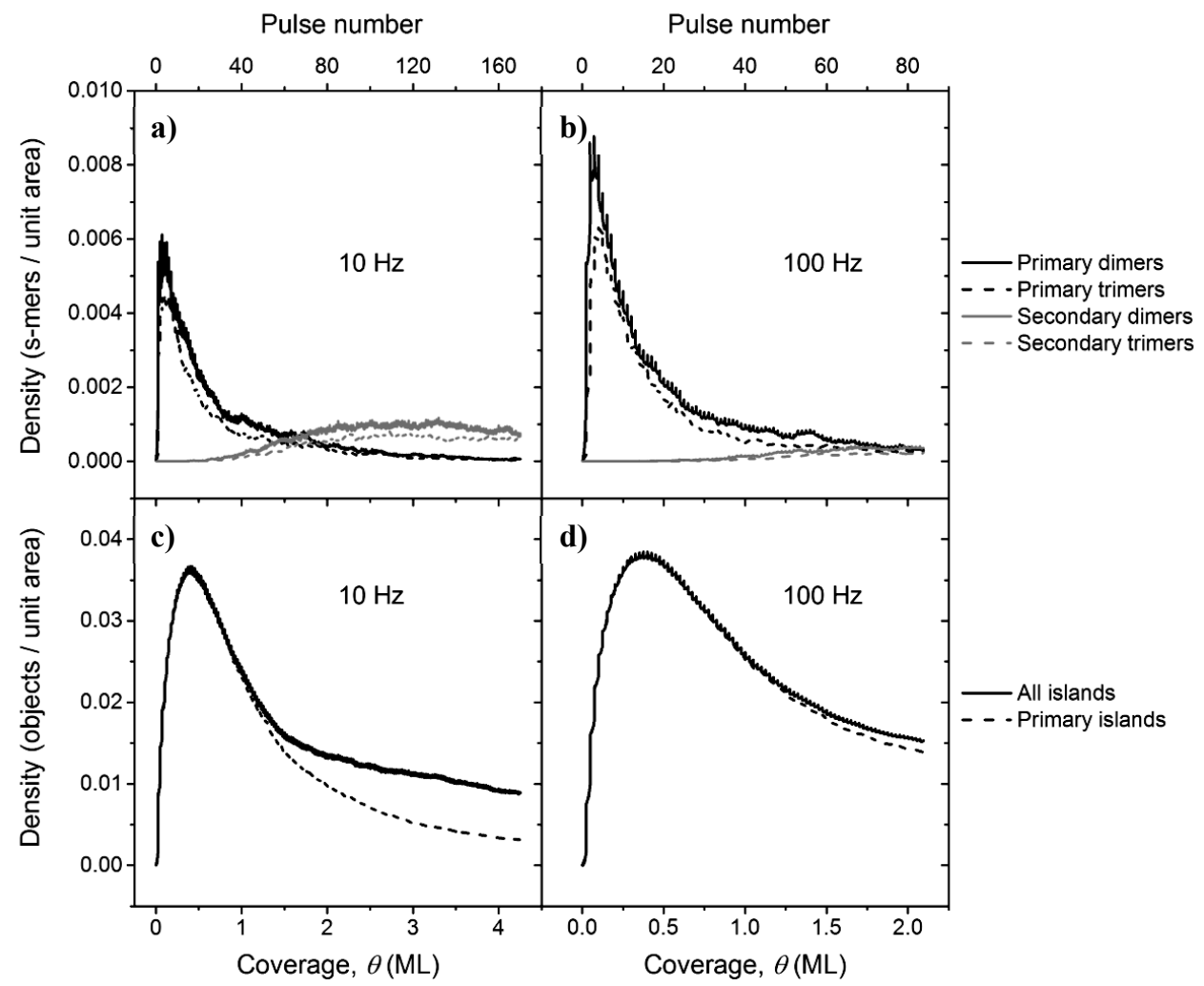

Figure 20. a), b) Number of primary and secondary dimers and trimers for depositions at $10 \mathrm{~Hz}$ and $100 \mathrm{~Hz}$. b), d) A comparison between island densities with and without secondary islands for depositions at $10 \mathrm{~Hz}$ and $100 \mathrm{~Hz} . D=858 \mathrm{a}^{2} / \mathrm{s}$, simulation box size $512 \times 512 a^{2}, F_{p}=$ $0.025 \mathrm{ML} /$ pulse $t_{\text {on }}=100 \mu \mathrm{s}$. 
Chapter 3 : Kinetic Monte CARLo Simulations 


\section{EXPERIMENTAL TECHNIQUES}

\subsection{DEPOSITION}

\subsubsection{MAgNeTRON SPUTTERING}

Film deposition is typically performed inside a vacuum chamber, where a material source is placed opposite to a collecting substrate (see Figure 21). [1] Magnetron sputtering is a physical vapor deposition (PVD) technique, in which the deposited particulates travel along a line-of-sight trajectory in contrast to chemical methods. In order to generate a vapor of the deposit material, noble gas plasma (typically argon, Ar) can be used to induce ion bombardment knockout of the source material, hence the source is called a target. The plasma is generated by sending Ar gas into the chamber and applying a negative potential (typically several hundred volts) on the target. This repels randomly formed free electrons close to the target, which upon collisions with Ar atoms causes ionization and further electron generation. The latter effects cause an avalanche of Ar ionization which transforms the Ar gas into a

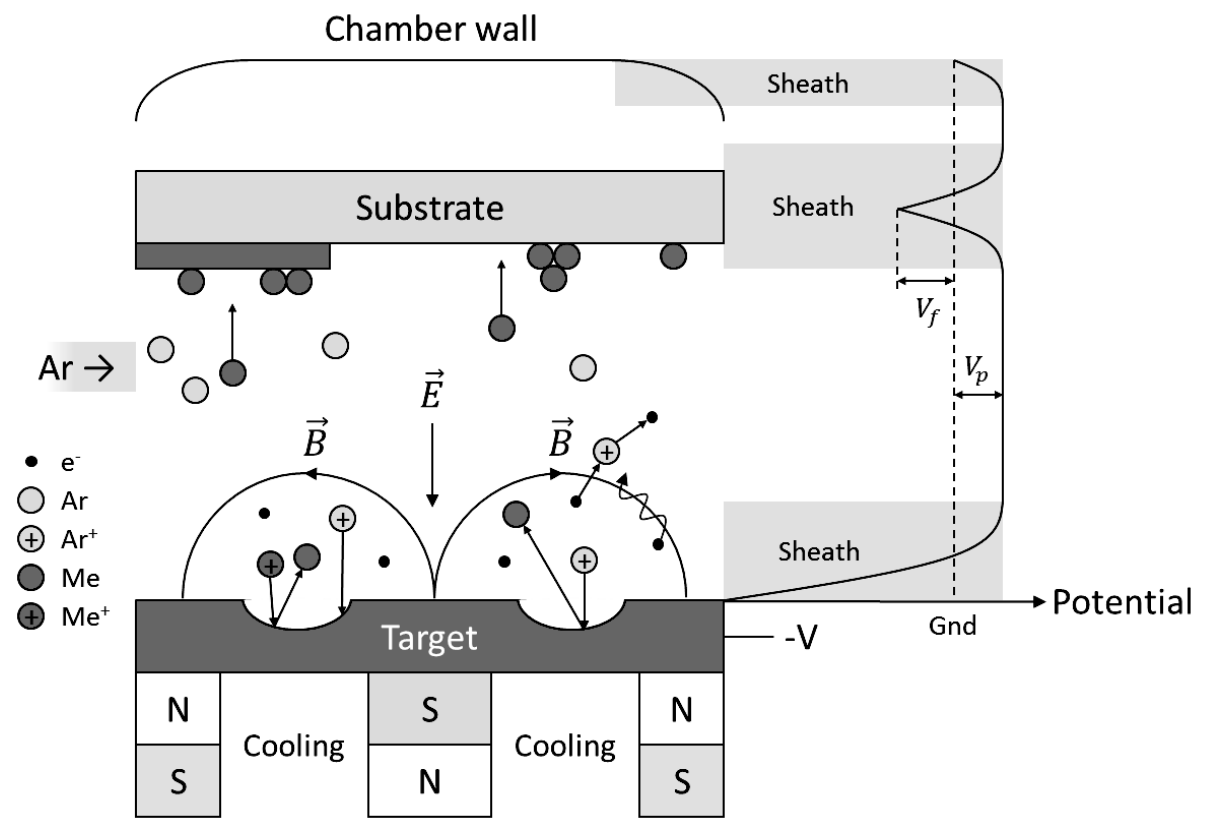

Figure 21. Schematic illustration of a magnetron sputtering process showing interactions between electrons ( $\left.\mathrm{e}^{-}\right)$, neutral $\mathrm{Ar}$ atoms $(\mathrm{Ar}), \mathrm{Ar}$ ions $\left(\mathrm{Ar}^{+}\right)$, target material atoms $(\mathrm{Me}$, assumed to be a metal) and target material ions $\left(\mathrm{Me}^{+}\right)$. The potential voltages on and around different objects are depicted in the graph to the right. 
quasi-neutral mix of ions and free electrons, i.e. plasma. Because Ar ions are positively charged, they are accelerated towards the target, and upon energy and momentum transfer, target atoms are "sputtered" out towards the substrate in a concentrated plume. The sputtering efficiency is amplified by trapping electrons close to the target using permanent magnets, hence the name magnetron. The electrons are trapped in the magnetic field due to the Lorentz force, causing a local enhancement of Ar ionization. This in turn causes groove formation on the target where sputtering occurs preferentially, commonly known as race-tracks due to the shape they form on the target surface. Self-sputtering may also occur when target atoms become ionized and are attracted back to the target with sufficient energy and momentum. However for conventional magnetron sputtering, the sputtered flux is mainly made up of neutral atoms. [15]

As electrons are far more mobile than ions in the plasma, these tend to absorb into any surface surrounding the plasma, causing the plasma in a vacuum chamber to be positively charged with a potential $V_{p}$ and forming "sheath" regions close to surfaces where the potential drops from $V_{p}$. If the surface is not grounded, i.e. electrically floating, an electron density collects and saturates on this surface, causing it to become negatively charged with a potential $V_{f}$. This is commonly the case on the substrate surface. Due to the net loss of electrons at surfaces, new electrons must be continuously supplied to keep the plasma alive. This is provided by secondary electron emission from the target due to sputtering.

\subsubsection{PUlSed VAPOR DEPOSITION}

Figure 22 illustrates schematically how pulsed power can be implemented experimentally for magnetron sputtering. By supplying voltage to the target in pulses, higher powers can be used without risking damage to the target or magnets. Since sputtering tends to heat the target, it is allowed to cool off in the time periods between pulses. Higher sputtering powers may be desired due to its ability to enhance ionization of the target material, which in turn allows the sputtered material to be steered by using an electrical bias on the substrate. However, this enhanced ionization is also known to cause back-attraction of sputtered atoms towards the target with a net effect of lowering the deposition rate.

In the context of the present work, time-domain effects of a pulsed vapor flux are of more central interest, and it has been shown that the arrival of sputter material at the substrate indeed possesses the same pulsing characteristics as the power applied to the target. [16] 


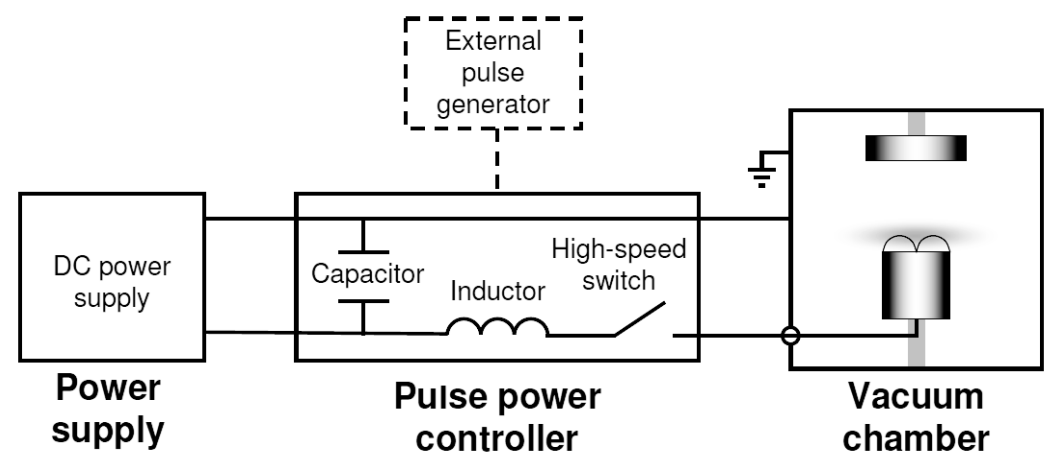

Figure 22. Schematic depiction of the experimental setup of pulsed magnetron sputtering adapted from reference [15]. A DC power supply is connected to the vacuum chamber via a pulse power controller. Within this controller, a capacitor bank is charged by the power supply, releasing the energy in pulses using high-speed switches.

\subsection{CHARACTERIZATION}

\subsubsection{SPECTROSCOPIC ELLIPSOMETRY}

With ellipsometry, the polarization state of light after reflection from (or transmission through) a material can be measured since the E-field of the incident light is polarized differently depending on its path through the material (see Figure 23). This is due to interactions between the electromagnetic waves and electrons and ions in the sample and can be characterized by the complex dielectric function of the material. Typically, it is used to gain information on optical properties, layer thicknesses, surface and interface roughness and

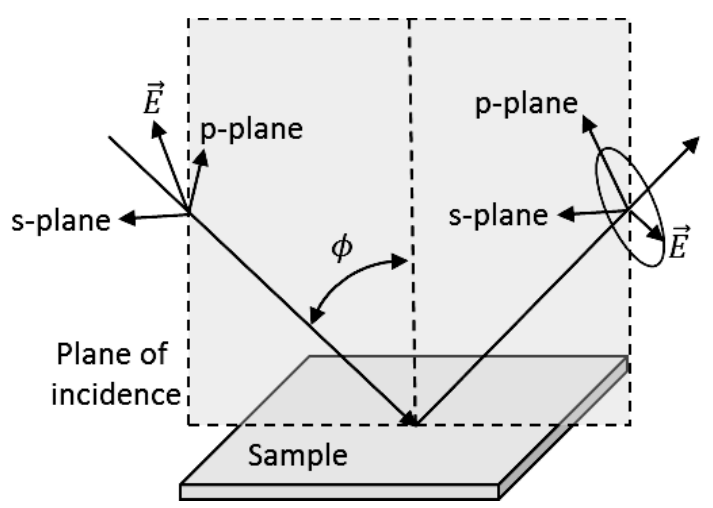

Figure 23. Schematic illustration of the interaction between a linearly polarized light beam and a sample in ellipsometry. $\phi$ is the variable incidence angle of the light beam. 
electrical properties. Owing to the relatively simple measurement setup and its non-intrusive nature, ellipsometry can be favorably used to perform in situ measurements during depositions.

In spectroscopic ellipsometry, a sample is irradiated with linearly polarized light of multiple energies ranging from the visible spectrum to the infra-red. After reflection at sample interfaces, this light generally becomes elliptically polarized. Quantitatively, this is represented by the complex ratio $\rho$ between the perpendicular $\left(r_{s}\right)$ and parallel $\left(r_{p}\right)$ components of the reflected light, which can be further parameterized by ellipsometry parameters as

$$
\rho=r_{p} / r_{s}=\tan (\Psi) \exp (i \Delta)
$$

where $\tan (\Psi)$ and $\Delta$ express the amplitude and phase of the complex number respectively. Depending on the physical aspects of a measured sample, it may be possible to find the dielectric function by direct inversion of the ellipsometry parameters $\Psi$ and $\Delta$. For a simple bulk material, an isotropic two-phase (air/material) model can be used

$$
\epsilon=\epsilon^{\prime}+i \epsilon^{\prime \prime}=\sin \left(\phi^{2}\right)\left[1+((1-\rho) /(1+\rho))^{2} \tan ^{2}(\phi)\right]
$$

where $\phi$ is the angle of incidence as shown in Figure 23. [151] This gives an exact solution to the dielectric function of the bulk material (for the measured energy range). For thin film samples, this is not as straight forward. A model needs to be created which takes into account all the different layers in a sample, from the substrate to the film through any eventual intermixed layers of these, as well as their various material properties. For each layer, a function is defined to model their individual contributions to the total dielectric function of the sample, the so called pseudo dielectric function. In the case of intermixed layers, an effective medium approximation (EMA) can be used which averages a set of dielectric functions, one for each constituent material. If vacuum or air (collectively known simply as 'void') is chosen as one of these constituent materials, the EMA can also be used to model surface roughness.

The dielectric function is related to the refractive index $n(\omega)$ and extinction coefficient $k(\omega)$ through

$$
\begin{gathered}
\epsilon^{\prime}(\omega)=n^{2}(\omega)-k^{2}(\omega) \\
\epsilon^{\prime \prime}(\omega)=2 n(\omega) k(\omega)
\end{gathered}
$$

These frequency dependent optical parameters and knowledge of the angles $\phi$ allow using the 
Fresnel equations to calculate model $r_{s, \text { calc }}(\omega)$ and $r_{p, \text { calc }}(\omega)$ values, which in turn yield $\rho_{\text {calc }}(\omega)$. By comparing $\rho_{\text {calc }}(\omega)$ to experimental values $\rho_{\text {exp }}(\omega)$ through a curve fitting procedure, model parameters providing the best match can be found. The final model parameters then represent the thin film material properties.

In this thesis, spectroscopic ellipsometry was used in Paper 1 and Paper 2 for in situ measurements of $\mathrm{Ag}$ thin films grown on $\mathrm{SiO}_{2}$ substrates during pulsed vapor deposition. The measurement data was then fitted by models described in the papers to find the time evolution of the areal coverage and thickness of films deposited under different conditions. From the fitting analysis, the percolation and continuous film formation thicknesses could be extracted in order to compare the scaling behaviors of these to the scaling behavior of the simulated elongation transition thicknesses.

\subsubsection{ATOMIC FORCE MICROSCOPY}

Atomic for microscopy (AFM) is often used to explore the topography of a surface. A small cantilever with an ideally atomic-dimensioned tip is traced along the surface of the film (see Figure 24). The tip is affected by forces (electrostatic or van der Waals) from the sample and the cantilever is bent accordingly. A laser the measures variations in the (vertical) position of the cantilever tip and translate these into an atomic height profile. Different modes of operation can be used with AFM. The simplest mode, known as contact mode, brings the tip close (atomic distances) to the sample surface and simply drags it along. This may however damage the sample and/or measuring tip, and non-contact operating modes were developed as

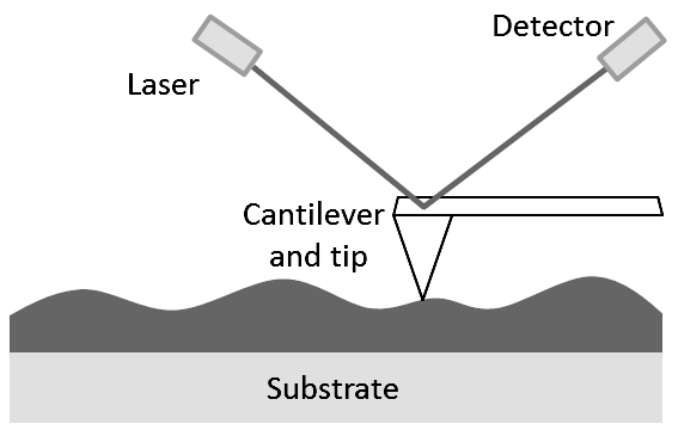

Figure 24. Schematic illustration of the principle of atomic force microscopy, where a tip mounted on a cantilever is traced along the sample surface, with a laser source and detector monitoring the movement of the cantilever due to atomic forces from the sample. 
well. In a non-contact mode, the tip is oscillated at or near its resonant frequency just above the sample surface. Forces from the sample acting on the tip tend to slow these oscillations, and the variations in the oscillation frequency can be interpreted into topographical information. Finally, if AFM is used under atmospheric conditions, the sample surface may be contaminated by liquid condensates, typically water. In this case, tapping mode can used, where the tip is alternately operated in contact (to cut through the condensates) and noncontact mode (to avoid damaging the sample).

In this thesis, AFM was used in Paper 1 to image the surface of films deposited at different pulse frequencies, in order to visualize the surface morphology around the percolation and continuous film formation stages of growth. 


\section{SUMMARY OF PAPERS}

Paper 1 investigates the effect of different pulsing frequencies on the growth evolution of $\mathrm{Ag}$ films, from nucleation to the formation of a continuous film by means of kMC simulations, in situ growth monitoring and ex situ imaging. Based on studies of the film thickness at characteristic growth transitions, it is shown that a pulsing frequency exists, below which the nominal transition thickness decreases with increasing pulsing frequency and above which the thickness stays constant. This behavior is determined to arise from the interplay between the pulsed deposition flux, as set by the pulsing frequency, and the time required for island coalescence to be completed within a cluster of islands.

Paper 2 compares simulated and experimental results of $\mathrm{Ag}$ films grown on $\mathrm{SiO}_{2}$ substrates under a continuous vapor flux. Specifically, the scaling behavior (with deposition rate) of the elongation transition thickness from $\mathrm{kMC}$ simulations are compared to the experimentally determined scaling behaviors of the percolation and continuous film formation transition thicknesses. Two growth regimes are identified in both simulations and experiments, which have been called the coalescence-controlled and coalescence-free growth regimes. The latter regime arises when coalescence is effectively hindered due to rapid lateral island growth caused by a combination of high deposition rate $F$ and substrate diffusivity $D$, for a given coalescence rate determined by the parameter $B$. Based on detailed studies of the rates of coalescence and island growth extracted from the $\mathrm{kMC}$ simulations, a theoretical framework is proposed that is able to predict the critical deposition rate $F_{c r i t}$ that for given $D$ and $B$ values will cause a crossover between the two growth regimes.

Paper 3 extends the theoretical treatment of Paper 2 to apply for pulsed vapor fluxes and also serves as an additional theoretical treatment of the results found in Paper 1. Since multiple deposition rate parameters exist for pulsed deposition that affect film nucleation in different ways depending on the temporal profile of the flux, three different expressions are derived for the critical frequency $f_{\text {crit }}$ (corresponding the $F_{\text {crit }}$ in the continuous case) that will cause a crossover from coalescence-controlled to coalescence-free growth regimes. Again these are functions of the substrate diffusivity $D$ and the coalescence rate parameter $B$, but also pulse parameters such as the pulse on-time $t_{o n}$ and the instantaneous deposition rate $F_{i}$ within a pulse. It is shown that these expressions each correspond to one of the three nucleation regimes typically found in pulsed vapor deposition. The theoretical results are compared to kMC simulations of pulsed vapor deposition for different combinations of the parameters 


\section{CHAPTER 5 : SUMMARY OF PAPERS}

mentioned thus far, i.e. $D, B, f, t_{o n}$ and $F_{i}$ with excellent agreement between theoretically predicted $f_{\text {crit }}$ values and simulated crossover values. 


\section{FUTURE OUTLOOK}

Plans are already in motion to begin developing a fully atomistic kMC simulation algorithm that will be used in particular to model binary metal alloy film growth on amorphous substrates. This will be coupled with ab initio or classical molecular dynamics simulations in order to fine-tune the inclusion of different atomic processes in the $\mathrm{kMC}$, as well as to provide values for different process activation barriers. It will be interesting to probe the dynamics of film growth when multiple timescales, attributed to the different materials, are in competition with each other and to investigate how to affect these by tuning the deposition process. These dynamics may cause exotic effects such as selective nucleation at seed sites, island shape selection, or capping of elemental islands of one material with the other. While technically more complex, fully atomistic simulations will allow for a deeper understanding of the coalescence process as it allows for the simulation of faceting as well as explicit surface diffusion on island surfaces. Also edge detachment can be modeled, which allows processes such as Ostwald ripening to be included. Irregular island shapes can be simulated, which allows the effects of angled deposition and competitive grain growth to be investigated as well.

Based on the understanding gained from the appended articles on polycrystalline growth of metal films on insulators or amorphous substrates by pulsed vapor deposition, new deposition strategies can be formulated to either tailor the film microstructure or to perform experimental measurements of various material properties. For the latter, the substrate diffusivity of various metals on amorphous substrates is of highest interest, since this is generally difficult to measure with more or less standardized techniques ${ }^{9}$. By determining what is called the "coalescence-free" growth regime in the appended papers for a specific film-substrate combination over a range of deposition rates and temperatures, it is believed $D$ can be calculated by using Eq. 8 .

Finally, using the knowledge in the articles, it may be possible to measure the elongation transition experimentally, albeit indirectly. Such measurements are of interest as they may be able to confirm the linear relationship of the film thickness at the elongation and percolation transitions. By producing template films of differing thicknesses under one set of conditions, film deposition may be continued on these templates under a different set of conditions. In

\footnotetext{
${ }^{9}$ Typically, STM is used to estimate the saturation island density, upon which the diffusivity can be backcalculated using Eq. 20 for known deposition rates. However, STM requires the substrate to be conductive and is thus not suitable for metal-on-insulator material systems.
} 
CHAPTER 6 : FUTURE OUTLOOK

this way, it becomes possible to estimate the elongation transition thickness based on how the continued film grows on a specific template. 


\section{REFERENCES}

[1] M. Ohring, Materials Science of Thin Films, 2nd Ed. (Academic press, 2001), p. 794.

[2] P. Jensen, Rev Mod Phys 71, 1695 (1999).

[3] L. Li, J. Hu, W. Yang, A. P. Alivisatos, M. Science, V. Di, and L. Berkeley, Nano Lett. 1, 349 (2001).

[4] I. Robel, M. Kuno, and P. V Kamat, J. Am. Chem. Soc. 129, 4136 (2007).

[5] T. Michely and J. Krug, Islands, Mounds, and Atoms: Patterns and Processes in Crystal Growth Far from Equilibrium (Springer Series in Surface Sciences) (Springer, 2003).

[6] R. Kern, G. Le Lay, and J. J. Metois, in Curr. Top. Mater. Sci. Vol. 3, edited by E. Kaldis (1979), pp. 131-420.

[7] J. W. Evans, P. a. Thiel, and M. C. Bartelt, Surf. Sci. Rep. 61, 1 (2006).

[8] H. Brune, Surf. Sci. Rep. 31, 125 (1998).

[9] I. Petrov, P. B. Barna, L. Hultman, and J. E. Greene, J. Vac. Sci. Technol. A Vacuum, Surfaces, Film. 21, S117 (2003).

[10] P. B. Barna and M. Adamik, Thin Solid Films 317, 27 (1998).

[11] J. E. Greene, in Handb. Depos. Technol. Film. Coatings, edited by P. M. Martin, 3rd Ed. (William Andrew Pub, Norwich, NY, 2010), pp. 554-620.

[12] N. Combe and P. Jensen, Phys. Rev. B 57, 15553 (1998).

[13] P. Jensen and B. Niemeyer, Surf. Sci. Lett. 384, L823 (1997).

[14] J. Warrender and M. Aziz, Phys. Rev. B 76, 045414 (2007).

[15] K. Sarakinos, J. Alami, and S. Konstantinidis, Surf. Coatings Technol. 204, 1661 (2010).

[16] D. Magnfält, V. Elofsson, G. Abadias, U. Helmersson, and K. Sarakinos, J. Phys. D. Appl. Phys. 46, 215303 (2013).

[17] E. Bauer, Int. Zeit. Krist. 110, 372 (1958).

[18] C. Kittel and K. Herbert, Thermal Physics (W. H. Freeman and Company, New York, 1980), pp. 275-308.

[19] W. K. Burton, N. Cabrera, and F. C. Frank, Philos. Trans. R. Soc. A Math. Phys. Eng. Sci. 243, 299 (1951).

[20] G. Ehrlich, J. Chem. Phys. 44, 1039 (1966).

[21] R. L. Schwoebel, J. Appl. Phys. 37, 3682 (1966).

[22] S. Stoyanov and I. Markov, Surf. Sci. 116, 313 (1982).

[23] J. Tersoff, A. W. D. van der Gon, and R. M. Tromp, Phys Rev Lett 72, 266 (1994).

[24] J. Rottler and P. Maass, Phys Rev Lett 83, 3490 (1999).

[25] J. Krug, P. Politi, and T. Michely, Phys. Rev. B 61, 37 (2000).

[26] J. Villain, J. Phys. I 1, 19 (1991).

[27] J. D. Weeks, in Ordering Strongly Fluctuating Condens. Matter Syst., edited by T. Riste (Springer US, 1980), pp. 293-317.

[28] G. S. Bales and D. C. Chrzan, Phys Rev Lett 74, 4879 (1995).

[29] G. S. Bales and D. C. Chrzan, Phys. Rev. B 50, 6057 (1994).

[30] E. Cox, M. Li, P.-W. Chung, C. Ghosh, T. Rahman, C. Jenks, J. Evans, and P. Thiel, Phys. Rev. B 71, 115414 (2005).

[31] M. C. Bartelt and J. W. Evans, Surf. Sci. 314, L829 (1994).

[32] Y. Li, M. C. Bartelt, J. W. Evans, N. Waelchli, E. Kampshoff, and K. Kern, Phys. Rev. B 56, 539 (1997).

[33] Y. Shim and J. Amar, Phys. Rev. B 73, 035423 (2006).

[34] J. Zhong, T. Zhang, Z. Zhang, and M. Lagally, Phys. Rev. B 63, 113403 (2001).

[35] Z. Zhang and M. G. Lagally, Science (80-. ). 276, 377 (1997).

[36] M. G. Lagally and Z. Zhang, Nature 417, 907 (2002).

[37] S. J. Liu, H. Huang, and C. H. Woo, Appl. Phys. Lett. 80, 3295 (2002).

[38] Y. Han, G.-H. Lu, B.-J. Lee, and F. Liu, Surf. Sci. 602, 2284 (2008).

[39] Y. Han, F. Liu, S.-C. Li, J.-F. Jia, Q.-K. Xue, and B.-J. Lee, Appl. Phys. Lett. 92, 021909 (2008).

[40] H. Yang, Q. Sun, Z. Zhang, and Y. Jia, Phys. Rev. B 76, 115417 (2007). 
[41] Z. Wang, Y. Li, and J. B. Adams, Surf. Sci. 450, 51 (2000).

[42] P. J. Feibelman, Surf. Sci. 478, L349 (2001).

[43] M. Schmid, E. Lundgren, G. Leonardelli, A. Hammerschmid, B. Stanka, and P. Varga, Appl. Phys. A 72, 405 (2001).

[44] E. Lundgren, B. Stanka, G. Leonardelli, M. Schmid, and P. Varga, Phys. Rev. Lett. 82, 5068 (1999).

[45] F. Rabbering, H. Wormeester, F. Everts, and B. Poelsema, Phys. Rev. B 79, 075402 (2009).

[46] H. Huang and J. Wang, Appl. Phys. Lett. 83, 4752 (2003).

[47] J. Wang, H. Huang, and T. S. Cale, Model. Simul. Mater. Sci. Eng. 12, 1209 (2004).

[48] R. Kunkel, B. Poelsema, L. K. Verheij, and G. Comsa, Phys Rev Lett 657, 733 (1990).

[49] M. R. Wilby and D. D. Vvedensky, Phys. Rev. B 47, 4119 (1993).

[50] B. D. Yu and M. Scheffler, Phys. Rev. B 56, 569 (1997).

[51] J. D. Wrigley and G. Ehrlich, Phys Rev Lett 44, 661 (1980).

[52] G. L. Kellogg and P. J. Feibelman, Phys Rev Lett 64, 3143 (1990).

[53] H. Bulou and C. Massobrio, Phys. Rev. B 72, 205427 (2005).

[54] H. Yildirim and T. S. Rahman, Phys. Rev. B 80, 235413 (2009).

[55] V. Stepanyuk, D. Bazhanov, W. Hergert, and J. Kirschner, Phys. Rev. B 63, 153406 (2001).

[56] J. Amar and F. Family, Phys. Rev. B. Condens. Matter 54, 14071 (1996).

[57] P. Jensen and X. Blase, Phys. Rev. B 70, 165402 (2004).

[58] F. Buatier de Mongeot, W. Zhu, a. Molle, R. Buzio, C. Boragno, U. Valbusa, E. Wang, and Z. Zhang, Phys. Rev. Lett. 91, 016102 (2003).

[59] K. A. Fichthorn and M. Scheffler, Nature 429, 617 (2004).

[60] M. Ozdemir and A. Zangwill, Phys. Rev. B 45, 3718 (1992).

[61] S. Tanaka, N. Bartelt, C. Umbach, R. Tromp, and J. Blakely, Phys. Rev. Lett. 78, 3342 (1997).

[62] S. N. Filimonov and Y. Y. Hervieu, Surf. Sci. 553, 133 (2004).

[63] B. S. Ranguelov and I. V. Markov, Cent. Eur. J. Phys. 7, 350 (2009).

[64] Y. Y. Hervieu and I. Markov, Surf. Sci. 628, 76 (2014).

[65] P. B. Barna and M. Adamik, in Sci. Technol. Thin Film., edited by F. C. Matacotta (1995), pp. $1-28$.

[66] V. Borovikov and J. Amar, Phys. Rev. B 72, 085460 (2005).

[67] J. Yu and J. Amar, Phys. Rev. B 69, 045426 (2004).

[68] J. Yu and J. Amar, Phys. Rev. Lett. 89, 286103 (2002).

[69] K. Caspersen and J. Evans, Phys. Rev. B 64, 075401 (2001).

[70] F. Montalenti and a. Voter, Phys. Rev. B 64, 081401 (2001).

[71] J. Seo, S.-M. Kwon, H.-Y. Kim, and J.-S. Kim, Phys. Rev. B 67, 121402 (2003).

[72] M. Li and J. Evans, Phys. Rev. Lett. 95, 256101 (2005).

[73] C. Bartelt and J. W. Evans, Phys. Rev. Lett. 75, 4250 (1995).

[74] P. Smilauer and D. Vvedensky, Phys. Rev. B 52, 14263 (1995).

[75] L. Ç. Arslan, C. Sanborn, E. Anzenberg, and K. F. Ludwig, Phys. Rev. Lett. 109, 106102 (2012).

[76] J. Amar and F. Family, Phys. Rev. Lett. 77, 4584 (1996).

[77] J. A. Venables, G. D. T. Spiller, and M. Hanbücken, Reports Prog. Phys. 47, 399 (1984).

[78] P. B. Barna, L. Tóth, and G. Vinczc, Surf. Coatings Technol. 57, 7 (1993).

[79] C. Polop, C. Rosiepen, S. Bleikamp, R. Drese, J. Mayer, A. Dimyati, and T. Michely, New J. Phys. 9, 74 (2007).

[80] S. Liu, Z. Zhang, and J. Nerskov, Surf. Sci. 321, 161 (1994).

[81] E. Vasco and J. Sacedón, Phys. Rev. Lett. 98, 036104 (2007).

[82] C. R. Stoldt, C. J. Jenks, P. a. Thiel, a. M. Cadilhe, and J. W. Evans, J. Chem. Phys. 111, 5157 (1999).

[83] M. Shen, J.-M. Wen, C. Jenks, P. Thiel, D.-J. Liu, and J. Evans, Phys. Rev. B 75, 245409 (2007).

[84] N. Combe, P. Jensen, and a Pimpinelli, Phys. Rev. Lett. 85, 110 (2000). 
[85] W. W. Mullins and G. S. Rohrer, J. Am. Ceram. Soc. 83, 214 (2000).

[86] A. La Magna, Surf. Sci. 601, 308 (2007).

[87] D. McCarthy and S. Brown, Phys. Rev. B 80, 064107 (2009).

[88] L. Lewis, P. Jensen, and J. Barrat, Phys. Rev. B 56, 2248 (1997).

[89] G. S. Rohrer, C. L. Rohrer, and W. W. Mullins, J. Am. Ceram. Soc. 104, 2099 (2001).

[90] D. Beysens, C. M. Knobler, and H. Schaffar, Phys. Rev. B 41, 9814 (1990).

[91] P. Meakin, Reports Prog. Phys. 55, 157 (1992).

[92] T. H. Lim, D. Mccarthy, S. C. Hendy, K. J. Stevens, S. A. Brown, and R. D. Tilley, ACS Nano 3, 3809 (2009).

[93] S. Hendy, S. Brown, and M. Hyslop, Phys. Rev. B 68, 241403 (2003).

[94] F. Ding, A. Rosén, and K. Bolton, Phys. Rev. B 70, 075416 (2004).

[95] M. Asoro, J. Damiano, and P. Ferreira, Microsc. Microanal. 15, 706 (2009).

[96] S. J. Zhao, S. Q. Wang, Z. Q. Yang, and H. Q. Ye, J. Physics. Condens. Matter 13, 8061 (2001).

[97] W. Luo, W. Hu, and S. Xiao, J. Phys. Chem. C 112, 2359 (2008).

[98] C. Herring, J. Appl. Phys. 21, 301 (1950).

[99] F. A. Nichols and W. W. Mullins, J. Appl. Phys. 36, 1826 (1965).

[100] G. C. Kuczynski, AIME Met. Trans. 185, 169 (1949).

[101] A. D. Brailsford and N. A. Gjostein, J. Appl. Phys. 46, 2390 (1975).

[102] W. W. Mullins, J. Appl. Phys. 28, 333 (1957).

[103] J. G. Amar, F. Family, and P.-M. Lam, Phys. Rev. B 50, 8781 (1994).

[104] M. Einax, S. Ziehm, W. Dieterich, and P. Maass, Phys. Rev. Lett. 99, 016106 (2007).

[105] M. C. Bartelt, M. C. Tringides, and J. W. Evans, Phys. Rev. B 47, 891 (1993).

[106] C. Ratsch, A. Zangwill, and P. Smilauer, Surf. Sci. Lett. 314, L937 (1994).

[107] F. Family and P. Meakin, Phys Rev Lett 61, 428 (1988).

[108] Y. A. Kryukov and J. G. Amar, Phys. Rev. B 81, 165435 (2010).

[109] J. Carrey and J.-L. Maurice, Phys. Rev. B 63, 245408 (2001).

[110] X. Yu, P. M. Duxbury, G. Jeffers, and M. A. Dubson, Phys. Rev. B 44, 13163 (1991).

[111] G. Jeffers, M. A. Dubson, and P. M. Duxbury, J. Appl. Crystallogr. 75, 5016 (1994).

[112] G. E. Pike and C. H. Seager, Phys. Rev. B 10, 1421 (1974).

[113] N. T. Liang, Y. Shan, and S. Wang, Phys. Rev. Lett. 37, 526 (1976).

[114] E. T. Gawlinski and H. E. Stanley, J. Phys. A. Math. Gen. 14, L291 (1981).

[115] D. Stauffer, Phys Rep 54, 1 (1979).

[116] M. Fanfoni and M. Tomellini, Phys. Rev. B. Condens. Matter 54, 9828 (1996).

[117] M. Fanfoni, M. Tomellini, and M. Volpe, Phys. Rev. B 64, 075409 (2001).

[118] M. Fanfoni, R. Polini, V. Sessa, M. Tomellini, and M. Volpe, Appl. Surf. Sci. 152, 126 (1999).

[119] M. Fanfoni and M. Tomellini, Appl. Surf. Sci. 136, 338 (1998).

[120] M. Kalff, P. Smilauer, G. Comsa, and T. Michely, Surf. Sci. 426, L447 (1999).

[121] J. Carrey and J.-L. Maurice, Phys. Rev. B 65, 205401 (2002).

[122] J. Carrey, J. Maurice, P. Jensen, and A. Vaures, Appl. Surf. Sci. 164, 48 (2000).

[123] I. Elkinani and J. Villain, J. Phys. I 4, 947 (1994).

[124] C. R. Henry, Prog. Surf. Sci. 80, 92 (2005).

[125] G. H. Gilmer, H. Huang, T. Diaz, D. Rubia, J. Dalla, and F. Baumann, Thin Solid Films 365, 189 (2000).

[126] S. Mahieu, P. Ghekiere, D. Depla, and R. De Gryse, Thin Solid Films 515, 1229 (2006).

[127] G. Antczak and G. Ehrlich, Surface Diffusion, 1st Ed. (Cambridge University Press, Cambridge, 2010).

[128] P. M. Agrawal, B. M. Rice, and D. L. Thompson, Surf. Sci. 515, 21 (2002).

[129] C. L. Liu, J. M. Cohen, J. B. Adams, and A. F. Voter, Surf. Sci. 253, 334 (1991).

[130] D. Walton, J. Chem. Phys. 37, 2182 (1962).

[131] M. V. Smoluchowski, Zeitschrift Fur Phys. 17, 557 (1916).

[132] G. Zinsmeister, Vacuum 529 (1965). 
[133] M. J. Stowell, J. Cryst. Growth 24/25, 45 (1974).

[134] J. W. Evans, J. Vac. Sci. Technol. A Vacuum, Surfaces, Film. 12, 1800 (1994).

[135] M. Popescu, J. Amar, and F. Family, Phys. Rev. B 58, 1613 (1998).

[136] G. S. Bales and a. Zangwill, Phys. Rev. B 55, R1973 (1997).

[137] H. Brune, G. S. Bales, J. Jacobsen, C. Boragno, and K. Kern, Phys. Rev. B 60, 5991 (1999).

[138] F. Family and P. Meakin, Phys. Rev. A 40, 3836 (1989).

[139] R. Vincent, Proc. R. Soc. A Math. Phys. Eng. Sci. 321, 53 (1971).

[140] B. J. Briscoe and K. P. Galvin, Phys. Rev. A 43, 1906 (1991).

[141] M. Körner, M. Einax, and P. Maass, Phys. Rev. B 82, 201401 (2010).

[142] M. Popescu, J. Amar, and F. Family, Phys. Rev. B 64, 205404 (2001).

[143] F. Shi, Y. Shim, and J. Amar, Phys. Rev. B 71, 245411 (2005).

[144] M. Aiempanakit, U. Helmersson, A. Aijaz, P. Larsson, R. Magnusson, J. Jensen, and T. Kubart, Surf. Coatings Technol. 205, 4828 (2011).

[145] A. C. Barato, H. Hinrichsen, and D. E. Wolf, Phys. Rev. E 77, 041607 (2008).

[146] J. Warrender and M. Aziz, Phys. Rev. B 75, 1 (2007).

[147] J. M. Warrender, Morphological Evolution of Nanocrystal Metal-on-Insulator Films Grown by Pulsed Laser Deposition, Harvard University, 2004.

[148] A. Chatterjee and D. G. Vlachos, J. Comput. Mater. Des. 14, 253 (2007).

[149] T. Schulze, Phys. Rev. E 65, 036704 (2002).

[150] A. F. Voter, in Radiat. Eff. Solids, edited by K. E. Sickafus and E. A. Kotomin (Springer, NATO Publishing Unit, Dordrecht, The Netherlands, 2005), pp. 1-23.

[151] H. G. Tompkins and E. A. Irene, editors, HANDBOOK OF ELLIPSOMETRY, 1st Ed. (William Andrew, Inc, Norwich, NY, 2005), pp. 1-902. 


\section{Appended Papers}

The articles associated with this thesis have been removed for copyright reasons. For more details about these see:

http://urn.kb.se/resolve?urn=urn:nbn:se:liu:diva-112136 